\title{
Benchmarking of objective quality metrics for HDR image quality assessment
}

\author{
Philippe Hanhart $^{1 *}$, Marco V. Bernardo ${ }^{2,3}$, Manuela Pereira ${ }^{3}$, António M. G. Pinheiro ${ }^{2}$ and Touradj Ebrahimi ${ }^{1}$
}

\begin{abstract}
Recent advances in high dynamic range (HDR) capture and display technologies have attracted a lot of interest from scientific, professional, and artistic communities. As in any technology, the evaluation of HDR systems in terms of quality of experience is essential. Subjective evaluations are time consuming and expensive, and thus objective quality assessment tools are needed as well. In this paper, we report and analyze the results of an extensive benchmarking of objective quality metrics for HDR image quality assessment. In total, 35 objective metrics were benchmarked on a database of $20 \mathrm{HDR}$ contents encoded with 3 compression algorithms at 4 bit rates, leading to a total of 240 compressed HDR images, using subjective quality scores as ground truth. Performance indexes were computed to assess the accuracy, monotonicity, and consistency of the metric estimation of subjective scores. Statistical analysis was performed on the performance indexes to discriminate small differences between metrics. Results demonstrated that metrics designed for HDR content, i.e., HDR-VDP-2 and HDR-VQM, are the most reliable predictors of perceived quality. Finally, our findings suggested that the performance of most full-reference metrics can be improved by considering non-linearities of the human visual system, while further efforts are necessary to improve performance of no-reference quality metrics for HDR content.
\end{abstract}

Keywords: Image quality assessment, Objective metrics, High dynamic range, JPEG XT

\section{Introduction}

Recently, the world of multimedia has been observing a growth in new imaging modalities aiming at improving user immersion capability, providing more realistic perception of content, and consequently, reaching new levels of quality of experience. This trend has began with the introduction of 3D capable devices in the consumer market, providing depth perception, followed by ultra high definition (UHD), focused on higher pixel resolutions beyond high definition, high frame rate (HFR), to provide more fluid motion, and, more recently, high dynamic range (HDR), intended to capture a wider range of luminance values. Moreover, academia, industry and service providers have proposed new models to further enrich content, such as plenoptic and holographic systems, although these latest modalities are still in very early stages. Many aspects need further improvement on such new trends. For instance, consumers still experience some

*Correspondence: philippe.hanhart@epfl.ch

1 Multimedia Signal Processing Group, EPFL, Lausanne, Switzerland

Full list of author information is available at the end of the article lack of reliable 3D content and still suffer from discomfort caused by long exposure. UHD systems face a small number of appropriate content although they have been evolving into consumer markets.

HDR imaging systems are stepping into the multimedia technologies for consumer market. HDR pursues a more complete representation of information that the human eye can see, capturing all the brightness information of the visible range of a scene, even in extreme lighting conditions. Hence, it pursues the representation of the entire dynamic range and color gamut perceived by human visual system (HVS). HDR imaging can be exploited to improve quality of experience in multimedia applications [1] and to enhance intelligibility in security applications where lighting conditions cannot be controlled [2].

HDR systems are becoming available for the general public. Acquisition systems are present in a large variety of photographic equipment and even in some mobile devices. Typically, computer rendering and merging of multiple low dynamic range (LDR) images taken at different exposure settings are the two methods used to generate HDR images [3]. Nowadays, HDR images can

\section{Springer}

(c) 2015 Hanhart et al. Open Access This article is distributed under the terms of the Creative Commons Attribution 4.0 International License (http://creativecommons.org/licenses/by/4.0/), which permits unrestricted use, distribution, and reproduction in any medium, provided you give appropriate credit to the original author(s) and the source, provide a link to the Creative Commons license, and indicate if changes were made. 
also be acquired using specific image sensors. HDR displays are also becoming increasingly available and enable representation of better contrasts, higher luminance, and wider color gamut [4]. Optionally, tone mapping operators (TMO) that map HDR content into the luminance range and color gamut of conventional displays can be used [5].

In addition to the acquisition and display technologies, JPEG has been standardizing new codecs for HDR content. JPEG XT is a recent standard for JPEG backwardcompatible compression of HDR images [6]. Using this compression standard, HDR images are coded in two layers. A tone-mapped version of the HDR image is encoded using the legacy JPEG format in a base layer, and the extra HDR information is encoded in a residual layer. The advantage of this layered scheme is that any conventional JPEG decoder can extract the tone-mapped image, keeping backward compatibility and allowing for display on a conventional LDR monitor. Furthermore, a JPEG XT compliant decoder can use the residual layer to reconstruct a lossy or even lossless version of the HDR image. Currently, JPEG XT defines four profiles (A, B, C, and D) for HDR image compression, of which profile $D$ is a very simple entry-level decoder that roughly uses the 12-bit mode of JPEG. Profiles A, B, and C all take into account the non-linearity of the human visual system. They essentially differ on the strategy used for creating the residual information and on the pre- and post-processing techniques. In profile $\mathrm{A}$, the residual is represented as a ratio of the luminance of the HDR image and the tone-mapped image after inverse gamma correction. The residual is log-encoded and compressed as an 8-bit greyscale image [7]. In profile $B$, the image is split into "overexposed" areas and LDR areas. The extension image is represented as a ratio of the HDR image and the tone-mapped image, after inverse gamma correction. Note that instead of a ratio, profile $\mathrm{B}$ uses a difference of logarithms. Finally, rofile $\mathrm{C}$ computes the residual image as a ratio of the HDR image and the inverse tone-mapped image. Unlike the other profiles, the inverse TMO is not a simple inverse gamma, but rather a global approximation of the inverse of the (possibly local) TMO that was used to generate the base-layer image. Similarly to profile $B$, the ratio is implemented as a difference of logarithms. However, instead of using the exact mathematical $\log$ operation, profile $\mathrm{C}$ uses a piecewise linear approximation, defined by re-interpreting the bit-pattern of the half-logarithmic IEEE representation of floatingpoint numbers as integers, which is exactly invertible [8]. MPEG is also starting a new standardization effort on HDR video [9], revealing the growing importance of HDR technologies.

As for any technology, evaluation of HDR systems, in terms of quality of experience, is essential. Subjective evaluations are time consuming and expensive, thus objective quality assessment tools are needed as well. To the best of our knowledge, only three objective metrics have been developed so far for HDR content. The most relevant work on this domain is the HDR visual detection predictor (HDR-VDP) metric proposed by Mantiuk et al. [10], which is an extension of Daly's VDP [11] for the HDR domain. The second version of this metric, HDR-VDP$2[12,13]$, is considered as the state-of-the-art in HDR image quality assessment. The dynamic range independent metric (DRIM) proposed in [14] can also be used for HDR quality assessment. Nevertheless, this metric results in three distortion maps, which is difficult to interpret, as there is no pooling of the different values. Recently, the high dynamic range video quality metric (HDR-VQM) was proposed by Narwaria et al. [15]. The metric was designed for quality assessment of HDR video content, but can also be used for HDR still images.

To overcome the lack of HDR objective metrics, LDR metrics, e.g., PSNR, were also used to evaluate HDR quality, especially in early HDR studies. However, LDR metrics are designed for gamma encoded images, typically having luminance values in the range $0.1-100 \mathrm{~cd} / \mathrm{m}^{2}$, while HDR images have linear values and are meant to capture a much wider range of luminance. Originally, gamma encoding was developed to compensate for the characteristics of cathode ray tube (CRT) displays, but it also takes advantage of the non-linearity in HVS to optimize quantization when encoding an image [16]. Under common illumination conditions, the HVS is more sensitive to relative differences between darker and brighter tones. According to Weber's law, the HVS sensitivity approximately follows a logarithm function for light luminance values [17]. Therefore, in several studies, LDR metrics have been computed in the log domain to predict HDR quality. However, at the darkest levels, the HVS sensitivity is closer to a square-root behavior, according to RoseDeVries law $[18,19]$. To extend the range of LDR metrics and to consider the sensitivity of the HVS, Aydin et al. [20] have proposed the perceptually uniform (PU) encoding. Another approach to apply LDR metrics on HDR images was proposed in [21]. This technique consists in tone-mapping the HDR image to several LDR images with different exposure ranges and to take the average objective score computed on each exposure. However, this approach is more time consuming and requires more computational power, proportionally to the number of exposures.

For LDR content, extensive studies have shown that not all metrics can be considered as reliable predictors of perceived quality [22,23], while only a few recent studies have benchmarked objective metrics for HDR quality assessment. The study of Valenzise et al. [24] compared the performance of PSNR and SSIM, computed in the logarithmic and PU [20] spaces, and HDR-VDP. The authors have concluded that non-uniformity must be corrected for 
a proper metric application, as most have been designed for perceptual uniform scales. Another subjective study was reported by Mantel et al. in [25]. A comparison with objective metrics in physical domain and using a gamma correction to approximate perceptually uniform luminance is also presented, concluding that the mean relative squared error (MRSE) metric provides the best performance in predicting quality. The correlation between 13 well-known full-reference metrics and perceived quality of compressed HDR content is investigated in [26]. The metrics were applied on the linear domain, and results show that only HDR-VDP-2 and FSIM predicted visual quality reasonably well. Finally, Narwaria et al. [15] have reported that their HDR-VQM metric performs similar or slightly better than HDR-VDP-2 for HDR image quality assessment. Regarding HDR video quality assessment, four studies were also reported by Azimi et al. [27], Rerabek et al. [28], Hanhart et al. [9], and Narwaria et al. [15]. The authors of [15] found that HDR-VQM is the best metric, far beyond HDR-VDP-2, in contradiction to the findings of [9], which showed lower performance for HDR-VQM when compared to HDR-VDP-2. Also, the other two studies found that HDR-VDP-2 has the highest correlation. The divergence between these findings might be due to the contents and types of artifacts considered in the different studies. Indeed, the first three studies consider HDR video sequences captured using HDR video cameras, manually graded, and encoded with compression schemes based on AVC or HEVC, whereas Narwaria et al. have mostly used computer-generated contents, an automatic algorithm to adjust the luminance of the HDR video sequences, and their own backward compatible HDR compression algorithm.

The main limitation of these studies lies in the small number of images or video sequences used in their experiments, which was limited to five or six contents. Also, a proper adaptation of the contents to the HDR display and correction of the metrics for non-uniformity were not always considered. Therefore, in this paper, we report and analyze the results of an extensive benchmarking of objective quality metrics for HDR image quality assessment. In total, 35 objective metrics were benchmarked using subjective scores as ground truth. The database used in our experiments $[29,30]$ is composed of 20 different contents and a total of 240 compressed HDR images with corresponding subjective quality scores. The HDR images are adapted (resized, cropped, and tone-mapped using display-adaptive tone-mapping operator) to SIM2 HDR monitor. The objective metrics were computed in the linear, logarithmic, PU [20], and Dolby perceptual quantizer (PQ) [31] domains. Additionally, the metrics were computed both on the luminance channel alone and as the average quality score of the $Y, C_{b}$, and $C_{r}$ channels. For each metric, objective scores were fitted to subjective scores using logistic fitting. Performance indexes were computed to assess the accuracy, monotonicity, and consistency of the metrics estimation of subjective scores. Finally, statistical analysis was performed on the performance indexes computed from 240 data points to discriminate small differences between two metrics. Hence, with this study, we expect to produce a valid contribution for future objective quality studies on HDR imaging.

The remainder of the paper is organized as follows. The dataset and corresponding subjective scores used as ground truth are described in Section 2.1. The different metrics benchmarked in this study are defined in Section 2.2. In Section 2.3, the methodology used to evaluate the performance of the metrics is described. Section 3 provides a detailed analysis of the objective results and discusses the reliability of objective metrics. Finally, Section 4 concludes the paper.

\section{Methodology}

The results of subjective tests can be used as ground truth to evaluate how well objective metrics estimate perceived quality. In this paper, we use the publicly available dataset provided by Korshunov et al. [29, 30] to benchmark 35 objective metrics. This section describes in details the dataset, objective metrics, and performance analysis used in our benchmark.

\subsection{Dataset and subjective scores}

The dataset is composed of 20 HDR images with a resolution of $944 \times 1080$ pixels. The dataset contains scenes with architecture, landscapes, and portraits and is composed of HDR images fused from multiple exposure pictures, frames extracted from HDR video, and computergenerated images. Since publicly available HDR images are usually not graded, the images are adjusted for a SIM2 HDR monitor using a display-adaptive TMO [32] to map the relative radiance representation of the images to an absolute radiance and color space of the HDR monitor. These display-adapted images are then considered as original images and compressed with JPEG XT using profiles $\mathrm{A}, \mathrm{B}$, and $\mathrm{C}$. The base and extension layers chromasubsampling are set to $4: 2: 0$ and 4:4:4, respectively, while optimized Huffman coding is enabled for all implementations. For each content and profile, four different bit rates were selected, leading to a total of 240 compressed HDR images. Figure 1 shows tone-mapped versions of the images in the dataset, and Table 1 reports the dynamic range and key [33] characteristics of these images. The key is in the range $[0,1]$ and gives a measure of the overall brightness

$$
k e y=\frac{\log L_{\mathrm{avg}}-\log L_{\min }}{\log L_{\max }-\log L_{\min }}
$$




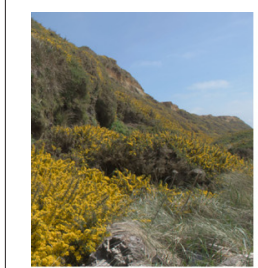

a BloomingGorse ${ }^{*}$

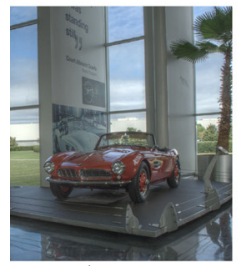

h $507^{*}$

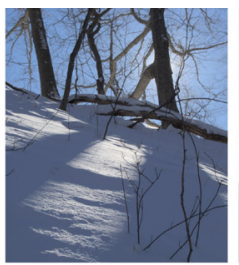

b DevilsBathtub*

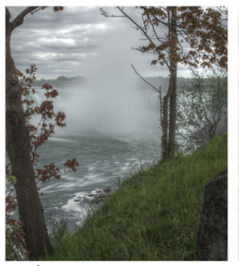

i CanadianFalls*

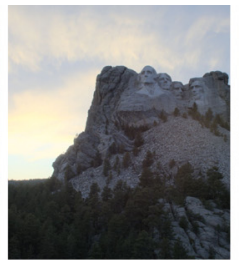

C MtRushmore 2*

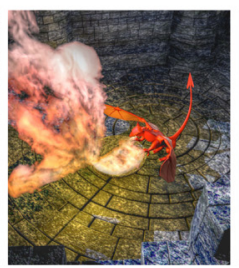

j dragon ${ }^{\#}$

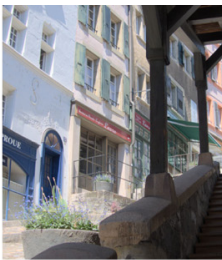

$\mathrm{d}_{\operatorname{set} 24}$

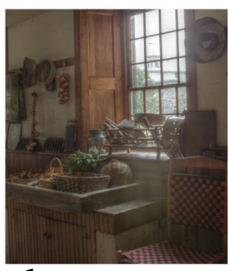

$\mathrm{K}_{\text {HancockKitchIn }}{ }^{*}$

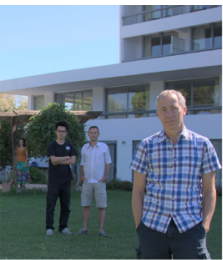

e $\operatorname{set} 70$

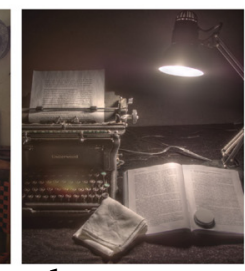

1 LabTypewriter ${ }^{*}$

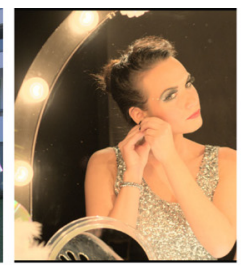

f showgirl

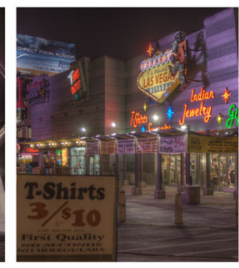

$\mathrm{m}_{\text {LasVegasStore }}$

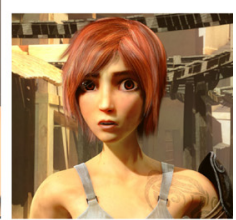

g sintel ${ }^{\dagger}$

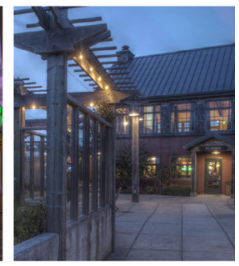

n McKeesPub*

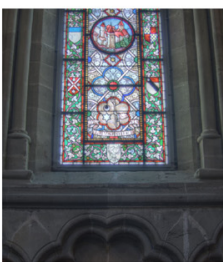

O $\operatorname{set} 18$

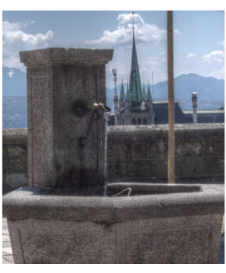

$\mathrm{P}_{\text {set } 22}$

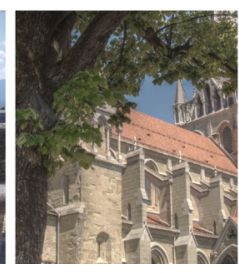

Q $\operatorname{set} 23$

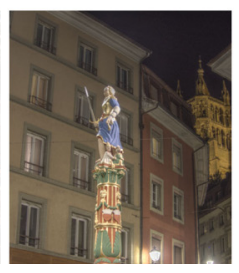

r set31

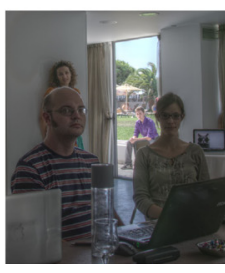

S set 33

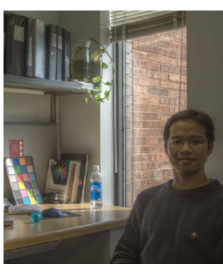

† WillyDesk*

Fig. 1 Display-adapted images of the dataset. The reinhard02 TMO was used for images from $\mathbf{a}-\mathbf{g}$ and the mantiuk06 TMO was used for the remaining images. Copyrights: *2006-2007 Mark D. Fairchild, †'Blender Foundation | www.sintel.org, under Creative Commons BY, \# Mark Evans, under Creative Commons BY

where $L_{\min }, L_{\max }$, and $L_{\text {avg }}$ are the minimum, maximum, and average luminance values, respectively, computed after excluding $1 \%$ of the darkest and lightest pixels.

The evaluation was performed using a full HD 47" SIM2 HDR monitor with individually controlled LED backlight modulation, capable of displaying content with luminance values ranging from 0.001 to $4000 \mathrm{~cd} / \mathrm{m}^{2}$. In every session, three subjects assessed the displayed test images simultaneously. They were seated in an arc configuration, at a constant distance of 3.2 times the picture height as recommended in [34], which corresponds to $1.87 \mathrm{~m}$ and a visual resolution of 60 pixels per degree. The laboratory was equipped with a controlled lighting system with a $6500 \mathrm{~K}$ color temperature, while a mid gray color was used for all background walls and curtains. The background luminance behind the monitor was set to $20 \mathrm{~cd} / \mathrm{m}^{2}$ and did not directly reflect off of the monitor.

The double-stimulus impairment scale (DSIS) Variant I methodology [35] was used for the evaluation. For scoring, a five-grade impairment scale (1: very annoying, 2: annoying, 3: slightly annoying, 4: perceptible, but not annoying, 5: imperceptible) was used. Two images were presented in side-by-side fashion with 32 pixels of black border separating the two images: one of the two images was always the reference (unimpaired) image, while the other was the test image. To reduce the effect of order of images on the screen, the participants were divided into two groups: the left image was always the reference image for the first group, whereas the right image was always the reference image for the second group. After the presentation of each pair of images, a six-second voting time followed. Subjects were asked to rate the impairments of the test images in relation to the reference image.

Before the experiment, oral instructions were provided to the subjects to explain their tasks. Additionally, a training session was organized, allowing subjects to familiarize themselves with the test procedure. For this purpose two images outside of the dataset were used. Five samples were manually selected by expert viewers for each image so that the quality of samples was representative of the rating scale.

Since the total number of test samples was too large for a single test session, the overall experiment was split into three sessions of approximately 16 min each. Between the sessions, subjects took a 15-min break. The test material was randomly distributed over the test sessions. To reduce contextual effects, the order of displayed stimuli was randomized applying different permutations for each group of subjects, whereas the same content was never shown consecutively. 
Table 1 Characteristics of HDR images from the dataset

\begin{tabular}{lll}
\hline & Dynamic range & Key \\
\hline 507 & 4.097 & 0.743 \\
AirBellowsGap & 4.311 & 0.768 \\
BloomingGorse2 & 2.336 & 0.748 \\
CanadianFalls & 2.175 & 0.729 \\
DevilsBathtub & 2.886 & 0.621 \\
dragon & 4.386 & 0.766 \\
HancockKitchenlnside & 4.263 & 0.697 \\
LabTypewriter & 4.316 & 0.733 \\
LasVegasStore & 4.131 & 0.636 \\
McKeesPub & 3.943 & 0.713 \\
MtRushmore2 & 4.082 & 0.713 \\
PaulBunyan & 2.458 & 0.702 \\
set18 & 4.376 & 0.724 \\
set22 & 3.162 & 0.766 \\
set23 & 3.359 & 0.764 \\
set24 & 3.862 & 0.778 \\
set31 & 4.118 & 0.678 \\
set33 & 4.344 & 0.698 \\
set70 & 3.441 & 0.735 \\
showgirl & 4.369 & 0.723 \\
sintel & 3.195 & 0.781 \\
WillyDesk & 4.284 & 0.777 \\
Min & 2.175 & 0.7821 \\
Max & 4.386 \\
Mean & & \\
Median & & \\
\hline
\end{tabular}

A total of 24 naïve subjects (12 females and 12 males) took part in the experiments. Subjects were aged between 18 and 30 years old with an average of 22.1. All subjects were screened for correct visual acuity and color vision using Snellen and Ishihara charts, respectively.

The subjective scores were processed by first detecting and removing subjects whose scores deviated strongly from others. The outlier detection was applied to the set of results obtained from the 24 subjects and performed according to the guidelines described in Section 2.3.1 of Annex 2 of [35]. Two outliers were detected. Then, the mean opinion score (MOS) was computed for each test stimulus as the mean score across the 22 valid subjects, as well as the associated $95 \%$ confidence interval (CI), assuming a Student's $t$-distribution of the scores. As it can be observed in Fig. 2, MOS values reflect the subjects perception fairly with enough MOS samples for each meaningful value range. More details about the dataset and subjective evaluations can be found in [29].

\subsection{Objective quality metrics}

Depending on the amount of information required about the reference image, objective metrics can be classified into three categories:

i) Full-reference (FR) metrics, which compare the test image with a reference image

ii) Reduced-reference (RR) metrics, which have access to a number of features from the reference image, extract the same features from the test image and compare them

iii) No-reference (NR) metrics, which do not use any information about the reference image

In this study, only FR and NR metrics were considered.

\subsubsection{Full-reference metrics}

To the best of our knowledge, there are only two metrics for HDR quality assessment that have a publicly available implementation: (1) HDR-VDP: high dynamic range visible difference predictor $[10,12,13]$ and (2) HDR-VQM: an objective quality measure for high dynamic range video [15].

The original HDR-VDP metric [10] was the first metric designed for HDR content. It is an extension of the VDP model [11] that considers a light-adaptive contrast sensitivity function (CSF), which is necessary for HDR content as the ranges of light adaptation can vary substantially. The metric was further extended [12] with different features, including a specific model of the point spread function (PSF) of the optics of the eye, as human optical lens flare can be very strong in high contrast HDR content. The front-end amplitude non-linearity is based on integration of the Weber-Fechner law. HDR-VDP is a calibrated metric and takes into account the angular resolution. The metric uses a multi-scale decomposition. A neural noise block is defined to calculate per-pixel probabilities maps of visibility and the predicted quality metric. In this study, we used the latest version of HDR-VDP, i.e., version 2.2.1 [13], referred to as HDR-VDP-2 in this paper.

HDR-VQM was designed for quality assessment of HDR video content. The metric is computed in the PU space and relies on a multi-scale and multi-orientations analysis, similarly to HDR-VDP, based on a subband decomposition using log-Gabor filters to estimate the subband errors. The subband errors are pooled over nonoverlapping spatiotemporal tubes to account for shortterm memory effects. Further spatial and long-term temporal poolings are performed to compute the overall quality score. In the case of still images, only spatial pooling is performed.

The remaining FR metrics considered in this study are all designed for LDR content and can be divided into different categories: difference measures and statisticaloriented metrics, structural similarity measures, visual 


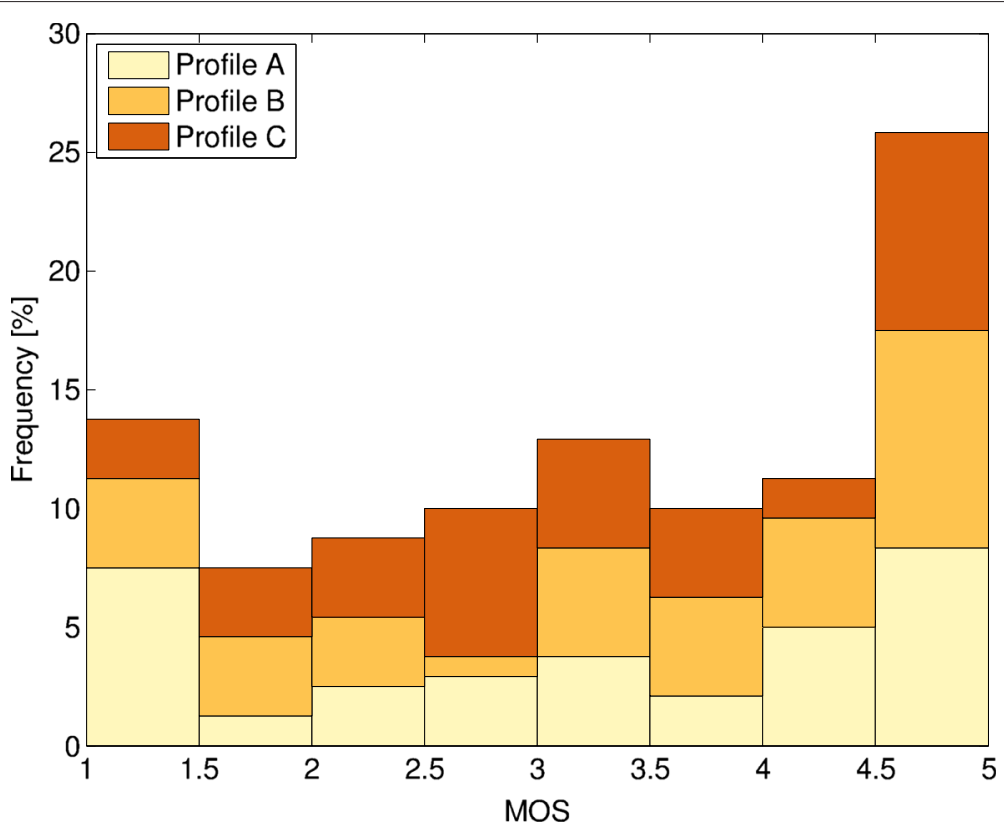

Fig. 2 MOS values distribution

information measures, information weighted metrics, HVS inspired metrics, and objective color difference measures (studied in the vision science). The complete list of considered FR metrics is provided in the following:

- Difference measures and statistical-oriented metrics These metrics are based on pixel color differences and provide a measure of the difference between the reference image and the distorted image. The following metrics of this category were considered: (3) MSE: mean squared error, (4) PSNR: peak signal-to-noise ratio, and (5) SNR: signal-to-noise ratio.

- Structural similarity measures

These metrics model the quality based on pixel statistics to model the luminance (using the mean), the contrast (variance), and the structure (crosscorrelation) [36]. The metrics considered in this category are the following: (6) UQI: universal quality index [37], (7) SSIM: structural similarity index [38], (8) MS-SSIM: multiscale SSIM index [39], (9) M-SVD: measure - singular value decomposition [40], and (10) QILV: quality index on local variance [41]. The MS-SSIM index is a multiscale extension of SSIM, which has a higher correlation with perceived quality when compared to SSIM. It is a perceptual metric based on the content features extraction and abstraction. This quality metric considers that the HVS uses the structural information from a scene
[38]. The structure of objects in the scene can be represented by their attributes, which are independent of both contrast and average luminance. Hence, the changes in the structural information from the reference and distorted images can be perceived as a measure of distortion. The MS-SSIM algorithm calculates multiple SSIM values at multiple image scales. By running the algorithm at different scales, the quality of the image is evaluated for different viewing distances. MS-SSIM also puts less emphasis on the luminance component when compared to contrast and structure components [39].

- Visual information measures

These metrics aim at measuring the image information by modeling the psycho-visual features of the HVS or by measuring the information fidelity. Then, the models are applied to the reference and distorted images, resulting in a measure of the difference between them. The following metrics on this category were considered: (11) IFC: image fidelity criterion [42], (12) VIF: visual information fidelity [43], (13) VIFp: VIF pixel-based version [43], and (14) FSIM: feature similarity index [44].

The VIF criterion analyses the natural scene statistics, using an image degradation model and the HVS model. This FR metric is based on the quantification of the Shannon information present in both the reference and the distorted images. VIFP is derived from the VIF criterion. 
FSIM is a perceptual metric that results from SSIM. FSIM adds the comparison of low-level feature sets between the reference and the distorted images [44]. Hence, FSIM analyzes the high phase congruency extracting highly informative features and the gradient magnitude, to encode the contrast information. This analysis is complementary and reflects different aspects of the HVS in assessing the local quality of an image.

- Information weighted metrics

The metrics in this category are based on the modeling of relative local importance of the image information. As not all regions of the image have the same importance in the perception of distortion, the image differences computed by any metrics have allocated local weights resulting in a more perceptual measure of quality. The following metrics were computed: (15) IW-MSE: information content weighting MSE [45], (16) IW-PSNR: information content weighting PSNR [45], and (17) IW-SSIM: information content weighting SSIM [45].

- HVS-inspired metrics These metrics try to model empirically the human perception of images from natural scenes. The following metrics were considered: (18) JND_st: just noticeable distortion [46], (19) WSNR: weighted SNR $[47,48]$, and (20) DN: divisive normalization [36].

- Objective color difference measures

The color difference metrics were developed because the CIE1976 color difference [49] magnitude in different regions of the color space did not appear correlated with perceived colors. These metrics were designed to compensate the non-linearities of the HVS present on the CIE1976 model. The following CIE metrics were computed: (21) CIE1976 [49], (22) CIE94 [50], (23) CMC [51], and (24) CIEDE2000 [52]. The CIEDE2000 metric is a color difference measure that includes not only weighting factors for lightness, chroma, and hue but also factors to handle the relationship between chroma and hue. The CIEDE2000 computation is not reliable in all color spaces. However, in this case, it can be used because the tested images are represented in the CIELAB color space that allows a precise computation.

\subsubsection{No-reference metrics}

These metrics are based on the analysis of a set of wellknown sharpness measures. The following NR metrics were considered: (25) JND: just noticeable distortion [46], (26) VAR: variance [53], (27) LAP: laplacian [54], (28) GRAD: gradient [54], (29) FTM: frequency threshold metric [55], (30) HPM: HP metric [56], (31) Marziliano: Marziliano blurring metric [55], (32) KurtZhang: kurtosisbased metric [57], (33) KurtWav: kurtosis of wavelet coefficients [58], (34) AutoCorr: auto correlation [54], and (35) RTBM: Riemannian tensor-based metric [59].

\subsubsection{Metrics computation and transform domains}

LDR metrics are designed for gamma encoded images, typically having luminance values in the range $0.1-100$ $\mathrm{cd} / \mathrm{m}^{2}$, while HDR images have linear values and are meant to capture a much wider range of luminance. Therefore, in this study, metrics were computed not only in the linear space but also in transformed spaces that provide a more perceptual uniformity. This space transformation was not applied to HDR-VDP-2 and HDR-VQM, which are calibrated metrics and require absolute luminance values as input. The color difference metrics, i.e., CIE1976, CIE94, CMC, and CIEDE2000, were also not computed in transformed spaces. These color difference measures require a conversion from the RGB representation to the CIELAB color space, considering a D65 $100 \mathrm{~cd} / \mathrm{m}^{2}$ reflective white point as reference white point.

Before any metric was computed, images were clipped to the range $[0.001,4000] \mathrm{cd} / \mathrm{m}^{2}$ (theoretical range of luminance values that the HDR monitor used in the subjective tests can render) to mimic the physical clipping performed by the HDR display. To compute the metrics in the linear domain, these luminance values were normalized to the interval $[0,1]$. This normalization was not applied to HDR metrics and to color difference metrics.

The remaining metrics were computed in three transform domains: the log domain, the PU domain [20], and the PQ domain [31]. The PU transform is derived using the threshold-integration method [60]. The transform is constrained such that luminance values in the range $0.1-80 \mathrm{~cd} / \mathrm{m}^{2}$, as produced by a typical CRT display, are mapped to the range $0-255$ to mimic the sRGB non-linearity. The PQ transform is derived from the Barten contrast sensitivity function [61]. The PQ curve has a square-root and log behavior at the darkest and highest light levels, respectively, while it exhibits a slope similar to the gamma non-linearities between those extreme luminance regions. Figure 3 depicts the normalized response of the log, PU, and PQ responses in the range $[0,4000] \mathrm{cd} / \mathrm{m}^{2}$.

These transformations were applied before any normalization and only after their application the resulting color components were normalized to the interval $[0,1]$. After the normalizations, the values considered to be in the RGB color space were transformed to the $Y C_{b} C_{r}$ color space [62]. The exception is the DN metric, which uses directly these RGB components. The metrics were computed on each of these components separately and two final metrics were considered: the quality score computed on the luminance channel alone and the average quality score of the $Y, C_{b}$, and $C_{r}$ channels. 

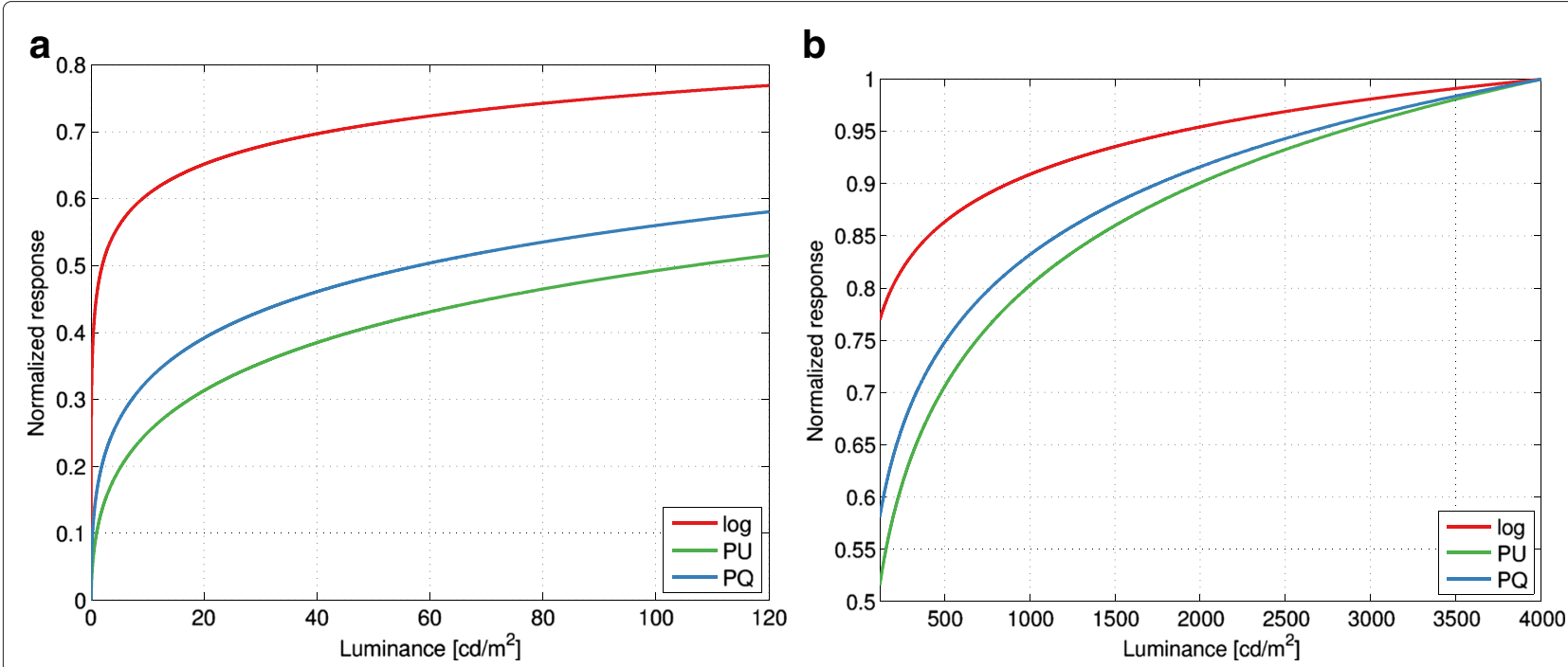

Fig. 3 Comparison of responses. Comparison of responses for the transformation functions in two different luminance ranges $\left(\mathrm{a}:[0,120] \mathrm{cd} / \mathrm{m}^{2}\right.$, b: $\left.[120,4000] \mathrm{cd} / \mathrm{m}^{2}\right)$

\subsection{Benchmarking of quality metrics}

To evaluate how well an objective metric is able to estimate perceived quality, the MOS obtained from subjective experiments are taken as ground truth and compared to predicted MOS values obtained from objective metrics. To compute the predicted MOS $\tilde{M}$, a regression analysis on each objective metric results $O$ was performed using a logistic function as a regression model:

$$
\tilde{M}=a+\frac{b}{1+\exp (-c \cdot(O-d))}
$$

where $a, b, c$, and $d$ are the parameters that define the shape of the logistic fitting function and were determined using a least squares method.

\subsubsection{Performance indexes}

Performance indexes to assess the accuracy of objective metrics were computed following the same procedure as in [63]. In particular, the Pearson linear correlation coefficient (PLCC) and the unbiased estimator of the rootmean-square error (RMSE) were used. The Spearman rank order correlation (SROCC) coefficient and the outlier ratio (OR) were also used to estimate respectively the monotonicity and the consistency of the objective metric as compared with the ground truth subjective data. The OR is the ratio of points for which the error between the predicted and actual MOS values exceeds the $95 \%$ confidence interval of MOS values.

\subsubsection{Statistical analysis}

To determine whether the difference between two performance index values corresponding to two different metrics is statistically significant, two-sample statistical tests were performed on all four performance indexes. In particular, for the PLCC and SROCC, a $Z$-test was performed using Fisher $z$-transformation. For the RMSE, an $F$-test was performed, whereas a $Z$-test for the equality of two proportions was performed for the OR. No processing was applied to correct for the multiple comparisons. The statistical tests were performed according to the guidelines of recommendation ITU-T P.1401 [64].

\section{Results}

Figures 4, 5, 6, and 7 report the accuracy, monotonicity, and consistency indexes, as defined in Section 2.3, for the metrics computed in the different domains. The metrics are sorted from best (top) to least (bottom) performing, based on the different performance indexes (higher PLCC/SROCC and lower RMSE/OR values indicate better performance). As HDR-VDP-2 and HDRVQM require absolute luminance values as input, these metrics were computed neither on the chrominance channels nor in the transform domains. Similarly, the different color difference metrics were computed only in the linear domain, after converting the absolute RGB values to the CIELAB color space. The DN metric was computed on the RGB components, considering all three channels together. Finally, the remaining 28 metrics were computed both on the luminance channel alone ( $Y$ suffix) and as the average quality score of the luminance, blue-difference, and red-difference channels ( $M$ suffix). The statistical analysis results are reported in the same tables. This analysis was performed on the performance indexes computed from 240 data points to discriminate small differences between two metrics. Metrics whose performance indexes are connected by a line are considered statistically not significantly different. For example, in the linear domain, 


\begin{tabular}{|c|c|c|c|c|c|c|c|}
\hline \multicolumn{2}{|l|}{ PLCC } & \multicolumn{2}{|l|}{ SROCC } & \multicolumn{2}{|l|}{ RMSE } & \multicolumn{2}{|l|}{ OR } \\
\hline HDRVDP2 & 0.9604 & HDRVDP2 & 0.9564 & HDRVDP2 & 0.3498 & HDRVDP2 & 0.3500 \\
\hline HDRVQM & 0.9602 & HDRVQM & 0.9564 & HDRVQM & 0.3506 & HDRVQM & 0.4083 \\
\hline IFC_Y & 0.9140 & IFC_Y & 0.9205 & IFC_Y & 0.5109 & IFC_Y & 0.5458 \\
\hline FSIM_Y & 0.8938 & FSIM_Y & 0.9160 & FSIM_Y & 0.5643 & UQI_Y & 0.5667 \\
\hline UQI_Y & 0.8873 & IFC_M & 0.8952 & UQI_Y & 0.5792 & IWPSNR_Y & 0.6167 \\
\hline IFC_M & 0.8855 & DN & 0.8926 & IFC_M & 0.5845 & IWSSIM_Y & 0.6167 \\
\hline DN & 0.8814 & WSNR_Y & 0.8791 & DN & 0.5936 & IWPSNR_M & 0.6417 \\
\hline WSNR_Y & 0.8786 & MSSSIM_Y & 0.8776 & WSNR_Y & 0.5995 & IWSSIM_M & 0.6417 \\
\hline FSIM_M & 0.8571 & FSIM_M & 0.8768 & FSIM_M & 0.6476 & IFC_M & 0.6458 \\
\hline MSSSIM_Y & 0.8545 & UQI_Y & 0.8737 & VIF_Y & 0.6521 & WSNR_Y & 0.6500 \\
\hline VIF_Y & 0.8545 & VIF_Y & 0.8617 & MSSSIM_Y & 0.6527 & VIF_Y & 0.6583 \\
\hline IWPSNR_Y & 0.8352 & IWMSE_Y & 0.8374 & IWPSNR_Y & 0.6907 & WSNR_M & 0.6667 \\
\hline IWSSIM_Y & 0.8352 & IWPSNR_Y & 0.8374 & IWSSIM_Y & 0.6907 & VIFP_Y & 0.6667 \\
\hline VIFP_Y & 0.8275 & IWSSIM_Y & 0.8374 & VIFP_Y & 0.7050 & DN & 0.6667 \\
\hline WSNR_M & 0.8178 & VIFP_Y & 0.8344 & WSNR_M & 0.7225 & FSIM_M & 0.6750 \\
\hline UQI_M & 0.8082 & IWMSE_M & 0.8298 & UQI_M & 0.7396 & FSIM_Y & 0.6833 \\
\hline IWPSNR_M & 0.8052 & WSNR_M & 0.8273 & IWPSNR_M & 0.7446 & UQI_M & 0.6917 \\
\hline IWSSIM_M & 0.8052 & IWPSNR_M & 0.8145 & IWSSIM_M & 0.7446 & CMC & 0.6917 \\
\hline CMC & 0.8045 & IWSSIM_M & 0.8145 & CMC & 0.7458 & VIF_M & 0.7000 \\
\hline CIE94 & 0.7987 & UQI_M & 0.8112 & CIE94 & 0.7558 & IWMSE_M & 0.7042 \\
\hline CIEDE00 & 0.7951 & CIE94 & 0.8031 & CIEDE00 & 0.7615 & MSSSIM_M & 0.7042 \\
\hline IWMSE_Y & 0.7951 & CMC & 0.8019 & IWMSE_Y & 0.7622 & CIEDE00 & 0.7083 \\
\hline IWMSE_M & 0.7907 & VIF_M & 0.7946 & IWMSE_M & 0.7689 & CIE94 & 0.7083 \\
\hline VIF_M & 0.7813 & CIEDE00 & 0.7908 & VIF_M & 0.7836 & SNR_M & 0.7208 \\
\hline MSVD_Y & 0.7629 & MSSSIM_M & 0.7877 & MSVD_Y & 0.8120 & SNR_Y & 0.7333 \\
\hline MSSSIM_M & 0.7610 & MSVD_Y & 0.7758 & MSSSIM_M & 0.8146 & PSNR_M & 0.7375 \\
\hline SNR_Y & 0.7535 & VIFP_M & 0.7657 & SNR_Y & 0.8253 & CIE1976 & 0.7458 \\
\hline VIFP_M & 0.7520 & MSVD_M & 0.7600 & VIFP_M & 0.8275 & VIFP_M & 0.7500 \\
\hline MSVD_M & 0.7466 & SNR_Y & 0.7574 & MSVD_M & 0.8355 & IWMSE_Y & 0.7542 \\
\hline SSIM_Y & 0.7374 & SSIM_Y & 0.7559 & SSIM_Y & 0.8482 & MSSSIM_Y & 0.7583 \\
\hline CIE1976 & 0.7254 & MSE_Y & 0.7262 & CIE1976 & 0.8642 & MSVD_M & 0.7667 \\
\hline PSNR_Y & 0.7152 & PSNR_Y & 0.7262 & PSNR_Y & 0.8774 & SSIM_M & 0.7708 \\
\hline MSE_Y & 0.7050 & CIE1976 & 0.7256 & MSE_Y & 0.8924 & PSNR_Y & 0.7792 \\
\hline SNR_M & 0.6872 & MSE_M & 0.6978 & SNR_M & 0.9120 & QILV_M & 0.7792 \\
\hline MSE_M & 0.6777 & SNR_M & 0.6969 & MSE_M & 0.9232 & MSVD_Y & 0.7833 \\
\hline PSNR_M & 0.6525 & PSNR_M & 0.6628 & PSNR_M & 0.9513 & MSE_M & 0.8000 \\
\hline QILV_Y & 0.6183 & QILV_Y & 0.6615 & QILV_Y & 0.9867 & QILV_Y & 0.8042 \\
\hline QILV_M & 0.6164 & QILV_M & 0.6535 & QILV_M & 0.9886 & SSIM_Y & 0.8125 \\
\hline SSIM_M & 0.5904 & SSIM_M & 0.5948 & SSIM_M & 1.0132 & MSE_Y & 0.8167 \\
\hline Marziliano_Y & 0.4551 & Marziliano_Y & 0.4160 & Marziliano_Y & 1.1178 & Marziliano_M & 0.8292 \\
\hline Marziliano_M & 0.3669 & HPM_M & 0.3341 & Marziliano_M & 1.1678 & Marziliano_Y & 0.8333 \\
\hline HPM_Y & 0.3625 & HPM_Y & 0.3211 & HPM_Y & 1.1700 & JND_St_M & 0.8375 \\
\hline HPM_M & 0.3503 & Marziliano_M & 0.3093 & HPM_M & 1.1758 & RTBM_Y & 0.8375 \\
\hline JND_St_Y & 0.2913 & JND_St_Y & 0.2393 & JND_St_Y & 1.2009 & RTBM_M & 0.8375 \\
\hline JND_St_M & 0.2509 & JND_St_M & 0.1599 & JND_St_M & 1.2152 & JND_St_Y & 0.8500 \\
\hline GRAD_M & 0.1060 & LAP_M & 0.1197 & GRAD_M & 1.2483 & VAR_Y & 0.8500 \\
\hline GRAD_Y & 0.1012 & LAP_Y & 0.1030 & GRAD_Y & 1.2489 & VAR_M & 0.8500 \\
\hline KurtZhang_M & 0.0829 & GRAD_M & 0.0784 & KurtZhang_M & 1.2513 & LAP_M & 0.8500 \\
\hline KurtWav_M & 0.0709 & GRAD_Y & 0.0742 & KurtWav_M & 1.2522 & GRAD_M & 0.8500 \\
\hline RTBM_Y & 0.0709 & KurtWav_M & 0.0607 & RTBM_Y & 1.2522 & FTM_Y & 0.8500 \\
\hline AutoCorr_Y & 0.0675 & KurtZhang_Y & 0.0518 & AutoCorr_Y & 1.2525 & HPM_Y & 0.8500 \\
\hline AutoCorr_M & 0.0664 & KurtZhang_M & 0.0366 & AutoCorr_M & 1.2526 & KurtZhang_Y & 0.8500 \\
\hline RTBM_M & 0.0623 & AutoCorr_Y & 0.0301 & RTBM_M & 1.2529 & KurtWav_Y & 0.8500 \\
\hline LAP_M & 0.0537 & AutoCorr_M & 0.0297 & LAP_M & 1.2535 & KurtWav_M & 0.8500 \\
\hline LAP_Y & 0.0458 & KurtWav_Y & 0.0265 & LAP_Y & 1.2540 & JND_Y & 0.8542 \\
\hline FTM_Y & 0.0324 & JND_M & 0.0138 & FTM_Y & 1.2547 & JND_M & 0.8542 \\
\hline KurtWav_Y & 0.0290 & JND_Y & 0.0110 & KurtWav_Y & 1.2548 & LAP_Y & 0.8542 \\
\hline JND_Y & 0.0200 & RTBM_Y & -0.0095 & JND_Y & 1.2551 & FTM_M & 0.8542 \\
\hline JND_M & 0.0198 & VAR_Y & -0.0221 & JND_M & 1.2551 & KurtZhang_M & 0.8542 \\
\hline KurtZhang_Y & 0.0194 & VAR_M & -0.0354 & KurtZhang_Y & 1.2551 & AutoCorr_Y & 0.8542 \\
\hline FTM_M & 0.0082 & FTM_Y & -0.0396 & FTM_M & 1.2553 & AutoCorr_M & 0.8542 \\
\hline VAR_M & 0.0068 & RTBM_M & -0.0421 & VAR_M & 1.2553 & GRAD_Y & 0.8625 \\
\hline VAR_Y & 0.0067 & FTM_M & -0.0748 & VAR_Y & 1.2553 & HPM_M & 0.8625 \\
\hline
\end{tabular}

Fig. 4 Accuracy, consistency, and monotonicity indexes for each objective metric computed in the linear space 


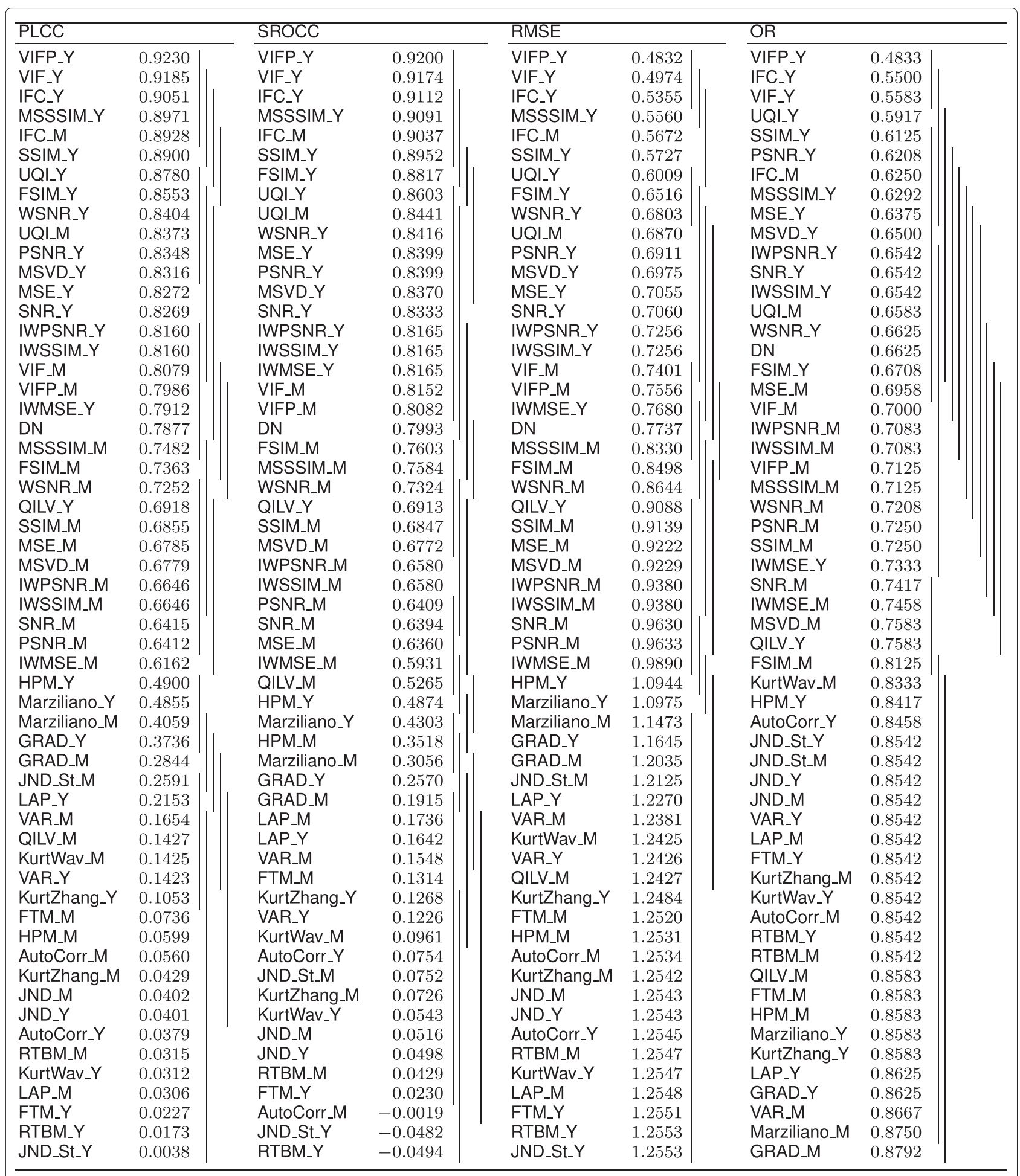

Fig. 5 Accuracy, consistency, and monotonicity indexes for each objective metric computed in the logarithm space

according to PLCC, there is no statistical evidence to show performance differences between IFC and FSIM computed on the luminance channel, but they are statistically different from HDR-VDP-2 (see Fig. 4).

\subsection{Best performing metrics}

As expected, HDR-VDP-2 and HDR-VQM, which are the only true HDR quality metrics considered in this study, computed on absolute luminance values, are the 


\begin{tabular}{|c|c|c|c|c|c|c|c|}
\hline \multicolumn{2}{|l|}{ PLCC } & \multicolumn{2}{|l|}{ SROCC } & \multicolumn{2}{|l|}{ RMSE } & \multicolumn{2}{|l|}{ OR } \\
\hline MSSSIM_Y & 0.9447 & MSSSIM_Y & 0.9501 & MSSSIM_Y & 0.4132 & $\overline{\text { VIF_Y }}$ & 0.4833 \\
\hline FSIM_Y & 0.9376 & FSIM_Y & 0.9470 & FSIM_Y & 0.4377 & IWPSNR_Y & 0.5167 \\
\hline VIF_Y & 0.9291 & VIF_Y & 0.9276 & VIF_Y & 0.4649 & IWSSIM_Y & 0.5167 \\
\hline VIFP_Y & 0.9288 & VIFP_Y & 0.9228 & VIFP_Y & 0.4656 & VIFP_Y & 0.5208 \\
\hline IWPSNR_Y & 0.9130 & IFC_Y & 0.9170 & IWPSNR_Y & 0.5121 & IFC_Y & 0.5375 \\
\hline IWSSIM_Y & 0.9130 & IWMSE_Y & 0.9109 & IWSSIM_Y & 0.5121 & MSSSIM_Y & 0.5417 \\
\hline IFC_Y & 0.9110 & IWPSNR_Y & 0.9109 & IFC_Y & 0.5196 & WSNR_Y & 0.5583 \\
\hline DN & 0.9078 & IWSSIM_Y & 0.9109 & DN & 0.5275 & FSIM_Y & 0.5625 \\
\hline SSIM_Y & 0.9060 & DN & 0.9090 & SSIM_Y & 0.5316 & SSIM_Y & 0.5792 \\
\hline WSNR_Y & 0.8959 & SSIM_Y & 0.9072 & WSNR_Y & 0.5577 & UQI_Y & 0.5833 \\
\hline IFC_M & 0.8928 & IFC_M & 0.9043 & IFC_M & 0.5670 & DN & 0.5875 \\
\hline IWMSE_Y & 0.8841 & WSNR_Y & 0.8950 & IWMSE_Y & 0.5878 & PSNR_Y & 0.5917 \\
\hline UQI_Y & 0.8777 & MSVD_Y & 0.8638 & UQI_Y & 0.6016 & SNR_Y & 0.5958 \\
\hline MSVD_Y & 0.8612 & UQI_Y & 0.8610 & MSVD_Y & 0.6392 & IWMSE_Y & 0.6250 \\
\hline PSNR_Y & 0.8526 & MSE_Y & 0.8564 & PSNR_Y & 0.6562 & IFC_M & 0.6375 \\
\hline SNR_Y & 0.8472 & PSNR_Y & 0.8564 & SNR_Y & 0.6669 & MSVD_Y & 0.6375 \\
\hline MSE_Y & 0.8352 & SNR_Y & 0.8556 & MSE_Y & 0.6915 & VIF_M & 0.6625 \\
\hline FSIM_M & 0.8310 & FSIM_M & 0.8484 & FSIM_M & 0.6991 & VIFP_M & 0.6667 \\
\hline UQI_M & 0.8278 & MSSSIM_M & 0.8442 & UQI_M & 0.7049 & UQI_M & 0.6833 \\
\hline VIF_M & 0.8275 & VIF_M & 0.8373 & VIF_M & 0.7053 & IWPSNR_M & 0.6917 \\
\hline MSSSIM_M & 0.8273 & UQI_M & 0.8335 & MSSSIM_M & 0.7059 & WSNR_M & 0.6917 \\
\hline VIFP_M & 0.8242 & VIFP_M & 0.8327 & VIFP_M & 0.7109 & IWSSIM_M & 0.6917 \\
\hline WSNR_M & 0.8163 & WSNR_M & 0.8233 & WSNR_M & 0.7254 & MSE_Y & 0.7000 \\
\hline IWPSNR_M & 0.7848 & QILV_Y & 0.8047 & IWPSNR_M & 0.7781 & QILV_M & 0.7042 \\
\hline IWSSIM_M & 0.7848 & IWPSNR_M & 0.7937 & IWSSIM_M & 0.7781 & SSIM_M & 0.7083 \\
\hline MSVD_M & 0.7837 & IWSSIM_M & 0.7937 & MSVD_M & 0.7804 & MSSSIM_M & 0.7083 \\
\hline QILV_Y & 0.7779 & MSVD_M & 0.7862 & QILV_Y & 0.7922 & IWMSE_M & 0.7458 \\
\hline IWMSE_M & 0.7414 & IWMSE_M & 0.7700 & IWMSE_M & 0.8426 & SNR_M & 0.7458 \\
\hline MSE_M & 0.7280 & MSE_M & 0.7444 & MSE_M & 0.8651 & FSIM_M & 0.7458 \\
\hline SSIM_M & 0.7194 & SSIM_M & 0.7324 & SSIM_M & 0.8719 & PSNR_M & 0.7542 \\
\hline SNR_M & 0.7085 & SNR_M & 0.7147 & SNR_M & 0.8859 & MSVD_M & 0.7542 \\
\hline PSNR_M & 0.7033 & PSNR_M & 0.7088 & PSNR_M & 0.8925 & QILV_Y & 0.7542 \\
\hline QILV_M & 0.6789 & QILV_M & 0.6739 & QILV_M & 0.9218 & MSE_M & 0.7708 \\
\hline Marziliano_Y & 0.5114 & HPM_Y & 0.4442 & Marziliano_Y & 1.0788 & HPM_Y & 0.8375 \\
\hline HPM_Y & 0.4548 & Marziliano_Y & 0.4179 & HPM_Y & 1.1181 & Marziliano_M & 0.8375 \\
\hline Marziliano_M & 0.4217 & HPM_M & 0.3679 & Marziliano_M & 1.1383 & JND_St_Y & 0.8417 \\
\hline HPM_M & 0.4004 & Marziliano_M & 0.3378 & HPM_M & 1.1503 & LAP_Y & 0.8417 \\
\hline JND_St_Y & 0.2975 & GRAD_Y & 0.2040 & JND_St_Y & 1.1985 & AutoCorr_M & 0.8458 \\
\hline LAP_Y & 0.1824 & GRAD_M & 0.1869 & LAP_Y & 1.2343 & RTBM_M & 0.8458 \\
\hline VAR_Y & 0.1736 & VAR_M & 0.1387 & VAR_Y & 1.2363 & VAR_Y & 0.8500 \\
\hline GRAD_M & 0.1618 & VAR_Y & 0.1258 & GRAD_M & 1.2389 & GRAD_M & 0.8500 \\
\hline GRAD_Y & 0.1599 & RTBM_Y & 0.1223 & GRAD_Y & 1.2397 & FTM_Y & 0.8500 \\
\hline VAR_M & 0.1031 & KurtZhang_Y & 0.1044 & VAR_M & 1.2487 & FTM_M & 0.8500 \\
\hline LAP_M & 0.0948 & LAP_M & 0.0858 & LAP_M & 1.2497 & AutoCorr_Y & 0.8500 \\
\hline RTBM_Y & 0.0946 & RTBM_M & 0.0744 & RTBM_Y & 1.2498 & JND_St_M & 0.8542 \\
\hline AutoCorr_Y & 0.0860 & LAP_Y & 0.0713 & AutoCorr_Y & 1.2507 & JND_Y & 0.8542 \\
\hline KurtZhang_Y & 0.0803 & KurtWav_M & 0.0634 & KurtZhang_Y & 1.2513 & JND_M & 0.8542 \\
\hline AutoCorr_M & 0.0609 & KurtWav_Y & 0.0596 & AutoCorr_M & 1.2530 & GRAD_Y & 0.8542 \\
\hline RTBM_M & 0.0577 & FTM_Y & 0.0578 & RTBM_M & 1.2533 & HPM_M & 0.8542 \\
\hline FTM_Y & 0.0560 & AutoCorr_Y & 0.0518 & FTM_Y & 1.2534 & KurtZhang_M & 0.8542 \\
\hline JND_St_M & 0.0545 & JND_M & 0.0515 & JND_St_M & 1.2538 & KurtWav_Y & 0.8542 \\
\hline KurtWav_M & 0.0422 & JND_Y & 0.0499 & JND_M & 1.2545 & KurtWav_M & 0.8542 \\
\hline JND_M & 0.0361 & KurtZhang_M & 0.0357 & JND_Y & 1.2545 & RTBM_Y & 0.8542 \\
\hline JND_Y & 0.0360 & AutoCorr_M & 0.0356 & KurtWav_Y & 1.2552 & LAP_M & 0.8625 \\
\hline KurtWav_Y & 0.0143 & JND_St_M & 0.0321 & KurtZhang_M & 1.2553 & KurtZhang_Y & 0.8625 \\
\hline KurtZhang_M & 0.0093 & JND_St_Y & 0.0313 & FTM_M & 1.2553 & VAR_M & 0.8667 \\
\hline FTM_M & 0.0090 & FTM_M & -0.0193 & KurtWav_M & 1.2553 & Marziliano_Y & 0.8708 \\
\hline
\end{tabular}

Fig. 6 Accuracy, consistency, and monotonicity indexes for each objective metric computed in the PU space

best performing metrics when compared to all other metrics and domains. Both metrics have a correlation above 0.95 and a particularly low RMSE (around 0.35) and low OR, whereas all other metrics have an OR above 0.48. HDR-VDP-2 (OR $=0.35$ ) has a slightly lower OR than HDR-VQM (OR = 0.4083), but there is no statistical evidence to show a significant difference. However, HDR-VQM is over three times faster than 


\begin{tabular}{|c|c|c|c|c|c|c|c|}
\hline \multicolumn{2}{|l|}{ PLCC } & \multicolumn{2}{|l|}{ SROCC } & \multicolumn{2}{|l|}{ RMSE } & \multicolumn{2}{|l|}{ OR } \\
\hline MSSSIM_Y & 0.9380 & MSSSIM_Y & 0.9435 & MSSSIM_Y & 0.4366 & VIF_Y & 0.4917 \\
\hline VIFP_Y & 0.9301 & FSIM_Y & 0.9361 & VIFP_Y & 0.4613 & VIFP_Y & 0.4958 \\
\hline VIF_Y & 0.9292 & VIF_Y & 0.9272 & VIF_Y & 0.4646 & IFC_Y & 0.5333 \\
\hline FSIM_Y & 0.9240 & VIFP_Y & 0.9242 & FSIM_Y & 0.4812 & IWPSNR_Y & 0.5458 \\
\hline SSIM_Y & 0.9107 & IFC_Y & 0.9151 & SSIM_Y & 0.5188 & IWSSIM_Y & 0.5458 \\
\hline IFC_Y & 0.9093 & SSIM_Y & 0.9117 & IFC_Y & 0.5243 & MSSSIM_Y & 0.5542 \\
\hline IWPSNR_Y & 0.9025 & IFC_M & 0.9039 & IWPSNR_Y & 0.5407 & WSNR_Y & 0.5750 \\
\hline IWSSIM_Y & 0.9025 & IWPSNR_Y & 0.9024 & IWSSIM_Y & 0.5407 & SNR_Y & 0.5792 \\
\hline IFC_M & 0.8930 & IWSSIM_Y & 0.9024 & IFC_M & 0.5667 & SSIM_Y & 0.5792 \\
\hline WSNR_Y & 0.8893 & IWMSE_Y & 0.9022 & WSNR_Y & 0.5743 & UQI_Y & 0.5875 \\
\hline DN & 0.8887 & DN & 0.8917 & DN & 0.5768 & PSNR_Y & 0.5917 \\
\hline UQI_Y & 0.8767 & WSNR_Y & 0.8890 & UQI_Y & 0.6039 & FSIM_Y & 0.6042 \\
\hline IWMSE_Y & 0.8730 & MSE_Y & 0.8656 & IWMSE_Y & 0.6132 & IWMSE_Y & 0.6250 \\
\hline MSVD_Y & 0.8604 & PSNR_Y & 0.8656 & PSNR_Y & 0.6400 & DN & 0.6375 \\
\hline PSNR_Y & 0.8603 & MSVD_Y & 0.8646 & MSVD_Y & 0.6409 & MSVD_Y & 0.6417 \\
\hline SNR_Y & 0.8511 & UQI_Y & 0.8603 & SNR_Y & 0.6592 & IFC_M & 0.6500 \\
\hline MSE_Y & 0.8451 & SNR_Y & 0.8589 & MSE_Y & 0.6721 & VIF_M & 0.6542 \\
\hline UQI_M & 0.8285 & MSSSIM_M & 0.8359 & UQI_M & 0.7037 & VIFP_M & 0.6583 \\
\hline VIF_M & 0.8282 & VIF_M & 0.8358 & VIF_M & 0.7039 & WSNR_M & 0.6833 \\
\hline VIFP_M & 0.8224 & UQI_M & 0.8344 & VIFP_M & 0.7143 & UQI_M & 0.6875 \\
\hline MSSSIM_M & 0.8181 & FSIM_M & 0.8315 & MSSSIM_M & 0.7224 & QILV_Y & 0.6917 \\
\hline FSIM_M & 0.8129 & VIFP_M & 0.8302 & FSIM_M & 0.7318 & QILV_M & 0.6917 \\
\hline WSNR_M & 0.8047 & WSNR_M & 0.8127 & WSNR_M & 0.7455 & MSE_M & 0.7083 \\
\hline QILV_Y & 0.7744 & QILV_Y & 0.7964 & QILV_Y & 0.7946 & IWPSNR_M & 0.7083 \\
\hline MSVD_M & 0.7671 & IWPSNR_M & 0.7734 & MSVD_M & 0.8058 & SSIM_M & 0.7083 \\
\hline IWPSNR_M & 0.7653 & IWSSIM_M & 0.7734 & IWPSNR_M & 0.8081 & IWSSIM_M & 0.7083 \\
\hline IWSSIM_M & 0.7653 & MSVD_M & 0.7690 & IWSSIM_M & 0.8081 & MSE_Y & 0.7167 \\
\hline SSIM_M & 0.7243 & IWMSE_M & 0.7368 & SSIM_M & 0.8655 & MSSSIM_M & 0.7292 \\
\hline MSE_M & 0.7219 & MSE_M & 0.7362 & MSE_M & 0.8688 & PSNR_M & 0.7417 \\
\hline IWMSE_M & 0.7125 & SSIM_M & 0.7359 & IWMSE_M & 0.8810 & SNR_M & 0.7417 \\
\hline SNR_M & 0.7041 & SNR_M & 0.7117 & SNR_M & 0.8914 & IWMSE_M & 0.7458 \\
\hline PSNR_M & 0.7007 & PSNR_M & 0.7088 & PSNR_M & 0.8956 & MSVD_M & 0.7500 \\
\hline QILV_M & 0.6601 & QILV_M & 0.6411 & QILV_M & 0.9430 & FSIM_M & 0.7708 \\
\hline Marziliano_Y & 0.5065 & HPM_Y & 0.4685 & Marziliano_Y & 1.0824 & LAP_Y & 0.8375 \\
\hline HPM_Y & 0.4717 & Marziliano_Y & 0.4199 & HPM_Y & 1.1069 & HPM_Y & 0.8375 \\
\hline Marziliano_M & 0.4213 & HPM_M & 0.3486 & Marziliano_M & 1.1385 & Marziliano_M & 0.8417 \\
\hline HPM_M & 0.4108 & Marziliano_M & 0.3267 & HPM_M & 1.1445 & AutoCorr_M & 0.8458 \\
\hline LAP_Y & 0.1929 & GRAD_Y & 0.2241 & LAP_Y & 1.2318 & RTBM_M & 0.8458 \\
\hline VAR_M & 0.1797 & GRAD_M & 0.1917 & VAR_M & 1.2349 & JND_St_Y & 0.8500 \\
\hline GRAD_Y & 0.1733 & VAR_M & 0.1483 & GRAD_Y & 1.2368 & FTM_Y & 0.8500 \\
\hline JND_St_Y & 0.1603 & VAR_Y & 0.1273 & GRAD_M & 1.2403 & HPM_M & 0.8500 \\
\hline GRAD_M & 0.1556 & RTBM_Y & 0.1177 & JND_St_Y & 1.2411 & JND_St_M & 0.8542 \\
\hline VAR_Y & 0.1048 & KurtZhang_Y & 0.1062 & VAR_Y & 1.2485 & JND_Y & 0.8542 \\
\hline LAP_M & 0.0978 & LAP_M & 0.1004 & LAP_M & 1.2493 & JND_M & 0.8542 \\
\hline KurtZhang_Y & 0.0867 & LAP_Y & 0.0846 & KurtZhang_Y & 1.2506 & GRAD_Y & 0.8542 \\
\hline AutoCorr_Y & 0.0724 & KurtWav_M & 0.0780 & AutoCorr_Y & 1.2521 & GRAD_M & 0.8542 \\
\hline RTBM_Y & 0.0651 & FTM_Y & 0.0642 & RTBM_Y & 1.2527 & FTM_M & 0.8542 \\
\hline KurtWav_M & 0.0587 & RTBM_M & 0.0639 & KurtWav_M & 1.2532 & KurtZhang_M & 0.8542 \\
\hline JND_Y & 0.0373 & AutoCorr_Y & 0.0578 & JND_Y & 1.2545 & KurtWav_Y & 0.8542 \\
\hline FTM_Y & 0.0372 & KurtWav_Y & 0.0546 & FTM_Y & 1.2545 & KurtWav_M & 0.8542 \\
\hline JND_M & 0.0362 & JND_M & 0.0511 & JND_M & 1.2545 & AutoCorr_Y & 0.8542 \\
\hline AutoCorr_M & 0.0333 & JND_Y & 0.0506 & AutoCorr_M & 1.2547 & RTBM_Y & 0.8542 \\
\hline RTBM_M & 0.0324 & KurtZhang_M & 0.0358 & RTBM_M & 1.2547 & VAR_M & 0.8583 \\
\hline KurtWav_Y & 0.0166 & JND_St_Y & 0.0310 & KurtWav_Y & 1.2552 & Marziliano_Y & 0.8583 \\
\hline JND_St_M & 0.0128 & AutoCorr_M & 0.0264 & JND_St_M & 1.2552 & KurtZhang_Y & 0.8625 \\
\hline KurtZhang_M & 0.0119 & JND_St_M & -0.0238 & KurtZhang_M & 1.2553 & LAP_M & 0.8667 \\
\hline FTM_M & -0.0086 & FTM_M & -0.0521 & FTM_M & 1.2553 & VAR_Y & 0.8708 \\
\hline
\end{tabular}

Fig. 7 Accuracy, consistency, and monotonicity indexes for each objective metric computed in the PQ space

HDR-VDP-2 [15], which makes it a suitable alternative to HDR-VDP-2.

The results for HDR-VDP-2 are in line with the finding of [26], slightly better than that of Valenzise et al. [24], but in contradiction with Mantel et al. [25], who reported a much lower correlation. However, Mantel et al. used unusual combinations of parameters for the base and extension layers, especially for content BloomingGorse. 
Narwaria et al. [15] found that HDR-VQM was performing significantly better than HDR-VDP-2 for both video and still image content. However, our results show that both metrics have similar performance, while it was reported in [9] that HDR-VQM performs lower than HDR-VDP-2 for HDR video compression. The divergence between these findings might be due to the contents and types of artifacts considered in the different studies.

In contrast to the HDR metrics, the NR metrics show the worst performance with PLCC and SROCC values below 0.5 and RMSE and OR values above 1 and 0.8 , respectively, independently of the domain in which the metric was computed. These results show that NR metrics are not sufficient to reach satisfactory prediction accuracy considering a perceptual domain and that specific NR metrics should be designed for HDR image quality assessment.

\subsection{Difference measures and statistical-oriented metrics}

Results show that MSE-based metrics, i.e., MSE, SNR, and PSNR, are not very reliable predictors of perceived quality when computed in the linear domain, with correlation between 0.65 and 0.75 . Higher PLCC values were reported in [26] for MSE and SNR (PLCC = 0.88), but the study was performed considering only five contents. These metrics are know to be very content dependent [65], which might explain the drop in performance when considering 20 images. The correlation of MSE-based metrics computed on the luminance channel alone can be improved by about 0.1 by considering a more perceptual domain than the linear domain, which does not take into account the contrast sensitivity response of the HVS. In the $\log$ and PU domains, the correlation is about 0.83 and 0.84 , respectively, which is in line with the results from [24]. Nevertheless, the performance of the MSEbased metrics computed as the average quality score of the $Y, C_{b}$, and $C_{r}$ channels did not improve when considering perceptual domains. These observations indicate that the log, PU, and PQ domains can better represent the luminance sensitivity of the HVS than the linear domain, but they might not be optimal for the chrominance sensitivity.

\subsection{Objective color difference measures}

In the linear domain, the color difference metrics, with the exception of the original CIE1976 color difference metric, are the best performing pixel-based metrics. They outperform the MSE-based metrics, but there is no statistical evidence to show a significant improvement over SNR computed on the luminance alone. Nevertheless, their correlation with perceived visual quality is only about $80 \%$, with an OR above $69 \%$, which cannot be considered as reliable prediction. Since the release of the CIE1976 color difference metric, two extensions have been developed in 1994 and 2000 to better address perceptual non-uniformities of the HVS. But, according to the benchmarking results, further improvements might be necessary for HDR images to handle non-uniformities in low and high luminance ranges, outside of the typical range of LDR displays. The color difference metrics are computed in the CIELAB color space, which considers relative luminance values with respect to a reference white point, typically a reflective D65 white point about 100 $120 \mathrm{~cd} / \mathrm{m}^{2}$. This reference white point is similar to the targeted peak luminance that is typically considered when calibrating LDR reference monitors. Therefore, for HDR images, one would be tempted to set the luminance of the reference white point considered in the color conversion equal to the peak luminance of the HDR monitor. However, this leads to lower performance of the color difference metrics and the reflective white point should also be used for HDR content instead.

\subsection{Structural similarity and visual information measures}

The performance of SSIM and its multiscale extension, MS-SSIM, is improved by considering logarithm instead of linear values and is even further improved by considering the $\mathrm{PU}$ or $\mathrm{PQ}$ transform. In particular, on the luminance channel, the correlation of SSIM is increased by about 0.15 from linear to logarithm, while MS-SSIM improved by only about 0.03 . From log to $\mathrm{PU} / \mathrm{PQ}$, improvements are relatively low for SSIM, whereas MS-SSIM exhibits a gain of about 0.04. Results show that MS-SSIM (luminance only) performs the best in PU and PQ spaces according to the PLCC, SROCC,

\begin{tabular}{|c|c|c|c|c|c|c|c|}
\hline \multicolumn{2}{|l|}{ PLCC } & \multicolumn{2}{|l|}{ SROCC } & \multicolumn{2}{|l|}{ RMSE } & \multicolumn{2}{|l|}{ OR } \\
\hline HDRVDP2 & 0.9604 & HDRVDP2 & 0.9564 & HDRVDP2 & 0.3498 & HDRVDP2 & 0.3500 \\
\hline HDRVQM & 0.9602 & HDRVQM & 0.9564 & HDRVQM & 0.3506 & HDRVQM & 0.4083 \\
\hline PU2MSSSIM_Y & 0.9447 & PU2MSSSIM_Y & 0.9501 & PU2MSSSIM_Y & 0.4132 & LOG_VIFP_Y & 0.4833 \\
\hline PQ2MSSSIM_Y & 0.9380 & PQ2MSSSIM_Y & 0.9435 & PQ2MSSSIM_Y & 0.4366 & PU2MSSSIM_Y & 0.5417 \\
\hline LOG_VIFP_Y & 0.9230 & LOG_VIFP_Y & 0.9200 & LOG_VIFP_Y & 0.4832 & PQ2MSSSIM_Y & 0.5542 \\
\hline LOG_PSNR_Y & 0.8348 & LOG_PSNR_Y & 0.8399 & LOG_PSNR_Y & 0.6911 & LOG_PSNR_Y & 0.6208 \\
\hline
\end{tabular}

Fig. 8 Statistical analysis comparing the HDR metrics and best performing metric of each domain 

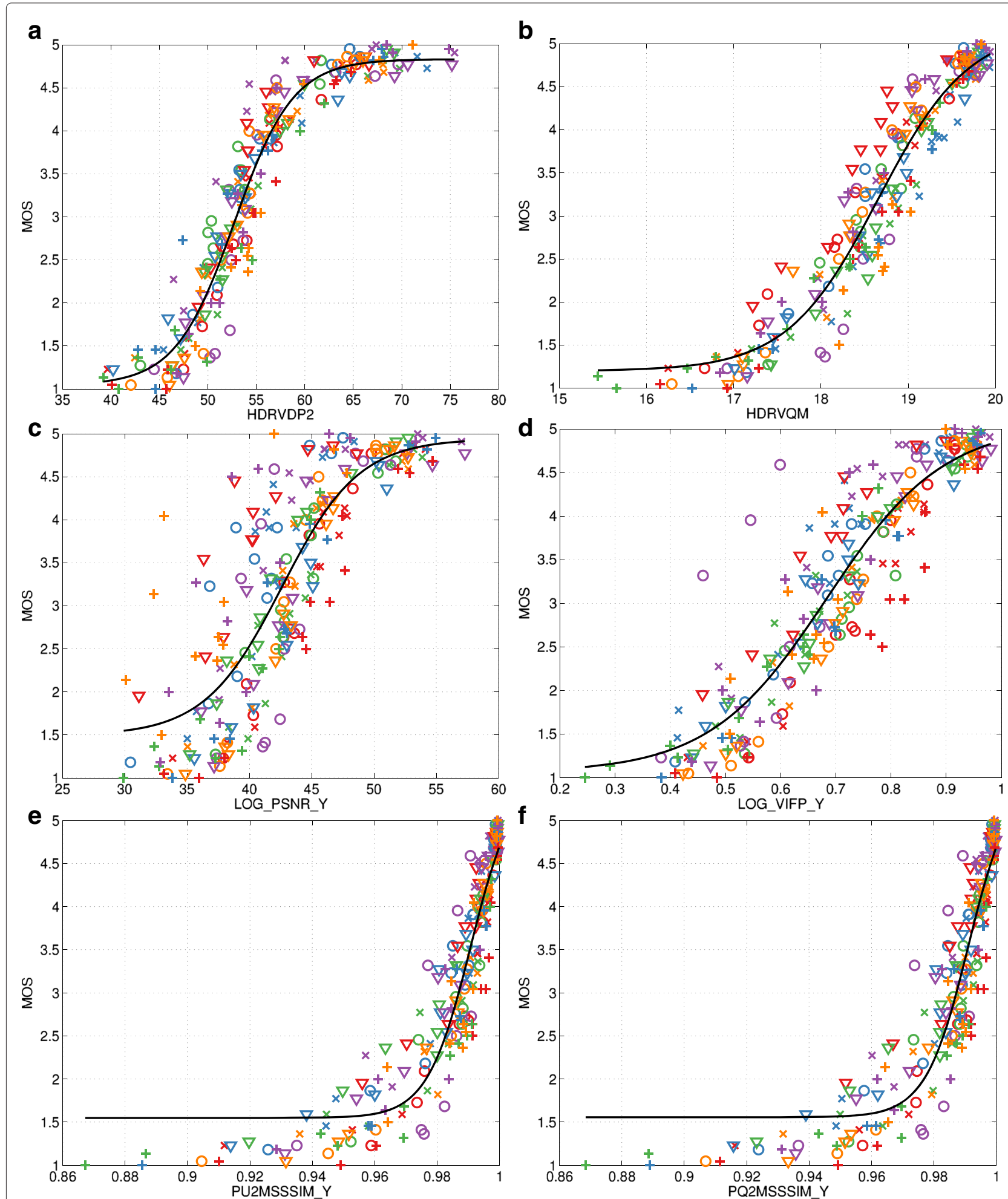

Fig. 9 Subjective versus objective results. Subjective versus objective evaluation results for the HDR metrics (a-b) and best performing metric of each domain (c-f). Each symbol, i.e., combination of marker and color, corresponds to a specific content 
and RMSE indexes. The correlation obtained for SSIM in the $\log$ and PU domains is similar to the results of Valenzise et al. [24]. On the other hand, UQI, which corresponds to the special case of SSIM when the constants $C_{1}$ and $C_{2}$ are set to 0 , does not perform better in the $\log$, PU, or PQ space than in the linear domain. Similar correlation results for SSIM and MS-SSIM are reported in [26] as in this paper (for the linear domain). However, it is reported that the relative change between the worst and best qualities for SSIM and MS-SSIM was less than 0.003 and $0.0003 \%$, respectively. In this study, the average relative change computed over all domains is 16.5 and $11.5 \%$ for SSIM and MS-SSIM, respectively. One major difference between the two works is the use of absolute luminance values in [26], whereas luminance values were linearly mapped from the theoretical display range to the range $[0,1]$ in this paper. For LDR content, SSIM uses different values for $C_{1}$ and $C_{2}$ depending on whether the images are in the range $[0,1]$ or $[0,255]$. For HDR content, our findings suggest that the value of these constants should be adjusted according the luminance range and depending on whether scaling of the values is performed or not.

Metrics that quantify the loss of image information, i.e., VIF, its pixel-based version, VIFP, and its predecessor, IFC, also show good performance. In particular, IFC (luminance only) is the second best performing metric in the linear domain. While the performance of IFC is not influenced by the domain in which the metric is computed, the performance of $\operatorname{VIF}(\mathrm{P})$ is significantly improved when considering a more perceptual domain than the linear space. In the log domain, results show that VIF computed on the luminance alone is the best performing metric. Note that the correlation reported for $\operatorname{VIF}(\mathrm{P})$ in this paper is significantly better than the one reported in [26]. Similarly to (MS-)SSIM, the difference might be due to the scaling procedure. Among the other HVS-based metrics, FSIM also shows good performance, especially in the PU and PQ space (RMSE below 0.5). In the linear domain, results are similar to our previous work.

\subsection{Statistical analyses}

To determine how the best metrics of each domain compare to each other, a direct benchmarking of the two HDR metrics, which are the best performing metrics in the linear space, and the best performing metric of the log, PU, and PQ spaces was performed. The PSNR metric computed on the luminance channel in the log space was added to this comparison, as this metric is widely used in HDR compression studies. Figure 8 reports the results of the statistical analysis of the six metrics. To identify metrics computed in the log, $\mathrm{PU}$, and $\mathrm{PQ}$ spaces, the $L O G_{-}$, $P U 2$, and $P Q 2$ prefixes are used, respectively. According to PLCC and SROCC, there is no statistical evidence to show performance differences between HDR-VDP-2, HDR$\mathrm{VQM}$, and MS-SSIM computed on the luminance channel in the PU space. However, HDR-VDP-2 and HDR-VQM have a significantly lower RMSE than all other metrics. Figure 9 depicts the scatter plots of subjective versus objective results for these metrics. As it can be observed, the data points are well concentrated near the fitting curve for HDR-VDP-2, as well as for HDR-VQM, while they are more scattered for the other metrics, especially in the case of LOG_PSNR_Y, which shows higher content dependency. These findings indicate that HDR-VDP-2 and HDR-VQM have a very high consistency when compared to the other metrics. Nevertheless, HDR-VDP-2 is complex and requires heavy computational resources, which limits its use in many applications. HDR-VQM and MS-SSIM computed in the PU space are lower complexity alternatives to HDR-VDP-2.

The statistical analysis was also used to understand whether there is a statistically significant difference between the performance of each metric when computed on the luminance component alone and when computed on all components. Only results from the analysis performed on the 28 metrics that were computed both on the $Y$ channel alone and as the average quality score of the $Y, C_{b}$, and $C_{r}$ channels were considered. Table 2 reports the number of metrics for which one approach was significantly better than the other one, as well as when no significant difference between the two approaches was observed. The analysis was performed individually for each performance index and domain. In the linear domain, there is no statistical evidence to show performance differences between the two approaches for about $80 \%$ of the metrics. However, in the log, PU, and PQ space, roughly half of the metrics perform significantly better

Table 2 Comparison of the 28 metrics computed on the $Y$ and $Y C_{b} C_{r}$ channels. Comparison of the metrics computed as the average quality score of the $Y$ channel alone and as the average quality score of the $Y C_{b} C_{r}$ channels

\begin{tabular}{|c|c|c|c|c|c|c|c|c|c|c|c|c|c|c|c|c|}
\hline & \multicolumn{4}{|l|}{$\operatorname{lin}$} & \multicolumn{4}{|l|}{$\log$} & \multicolumn{4}{|l|}{ PU } & \multicolumn{4}{|l|}{$\mathrm{PQ}$} \\
\hline & PLCC & SROCC & RMSE & OR & PLCC & SROCC & RMSE & OR & PLCC & SROCC & RMSE & OR & PLCC & SROCC & RMSE & OR \\
\hline$Y$ is better & 6 & 7 & 8 & 3 & 16 & 14 & 16 & 8 & 16 & 14 & 15 & 14 & 14 & 14 & 15 & 14 \\
\hline Similar & 22 & 21 & 20 & 25 & 11 & 14 & 12 & 20 & 12 & 14 & 13 & 14 & 14 & 14 & 13 & 14 \\
\hline$Y C_{b} C_{r}$ is better & 0 & 0 & 0 & 0 & 1 & 0 & 0 & 0 & 0 & 0 & 0 & 0 & 0 & 0 & 0 & 0 \\
\hline
\end{tabular}


Table 3 Comparison of the 57 metrics computed on all domains. Results represent the number of times a metric computed in the domain $i$ performs significantly better than when computed in the domain $j$, where $i$ and $j$ are the row and column of the table

\begin{tabular}{|c|c|c|c|c|c|c|c|c|c|c|c|c|c|c|c|c|}
\hline & \multicolumn{4}{|c|}{ PLCC } & \multicolumn{4}{|c|}{ SROCC } & \multicolumn{4}{|c|}{ RMSE } & \multicolumn{4}{|l|}{ OR } \\
\hline & $\operatorname{lin}$ & $\log$ & PU & $P Q$ & $\operatorname{lin}$ & $\log$ & PU & $P Q$ & $\operatorname{lin}$ & $\log$ & $\mathrm{PU}$ & $P Q$ & $\operatorname{lin}$ & $\log$ & PU & $P Q$ \\
\hline $\operatorname{lin}$ & 0 & 9 & 5 & 6 & 0 & 9 & 6 & 8 & 0 & 8 & 5 & 5 & 0 & 2 & 1 & 1 \\
\hline $\log$ & 10 & 0 & 2 & 2 & 10 & 0 & 4 & 4 & 8 & 0 & 0 & 0 & 8 & 0 & 3 & 3 \\
\hline PU & 14 & 17 & 0 & 11 & 13 & 15 & 0 & 11 & 11 & 16 & 0 & 10 & 9 & 7 & 0 & 7 \\
\hline$P Q$ & 13 & 17 & 8 & 0 & 11 & 15 & 8 & 0 & 11 & 16 & 6 & 0 & 9 & 7 & 3 & 0 \\
\hline
\end{tabular}

when computed on the luminance channel alone. According to PLCC, the JND metric, FR version, computed in the log domain, is the only case for which better performance is achieved when considering all channels. As HDR is often considered in combination with wide color gamut (WCG), it is expected that the fidelity of color reproduction will play a more important role in the context of HDR when compared to LDR. We believe that improvements can be achieved by considering different domains for computing the metrics on the chrominance channels and by using better pooling strategies.

Similarly, the statistical analysis was also used to understand whether there is a statistically significant difference between the performance of a particular metric computed in one domain and another domain. Only results from the analysis performed on the 57 metrics that were computed in all domains were considered. Table 3 reports the number of times a metric computed in the domain $i$ performs significantly better than when computed in the domain $j$, where $i$ and $j$ are the row and column of the table. Results show that most metrics perform the best in the PU and PQ spaces when compared to the lin and log spaces, which is in line with our previous observations. Note that results based on PLCC, SROCC, and RMSE are in agreement, while the OR metric shows fewer cases where statistically significant differences are observed. Additionally, there are also metrics for which computations performed in the linear and logarithm domains perform better than in the PU and PQ space. Overall, there is no optimal domain that performs the best for all metrics. Instead, different metrics should use different domains to maximize the correlation with perceived quality.

\section{Conclusions}

In this paper, 35 objective metrics were benchmarked on a database of 240 compressed HDR images using subjective quality scores as ground truth. Additionally to the linear space, metrics were computed in the logarithm, PU, and PQ domains to mimic non-linearities of the HVS. Results showed that the performance of most full-reference metrics can be improved by considering perceptual transforms when compared to linear values. On the other hand, our findings suggested that a lot of work remains to be done for no-reference quality assessment of HDR content. Our benchmark demonstrated that HDR-VDP-2 and HDR-VQM are ultimately the most reliable predictors of perceived quality. Nevertheless, HDR-VDP-2 is complex and requires heavy computational resources, which limits its use in many applications. HDR-VQM is over three times faster, which makes it a suitable alternative to HDR-VDP-2. Alternatively, MS-SSIM computed in the PU space is another lower complexity substitute, as there is no statistical evidence to show performance differences between these metrics in terms of PLCC and SROCC. Even though the numbers of contents and compressed images considered in the experiments are quite large, different performance might be observed for other contents and types of artifacts.

\section{Competing interests}

The authors declare that they have no competing interests.

\section{Acknowledgements}

This work has been conducted in the framework of the Swiss National Foundation for Scientific Research (FN 200021-143696-1), Swiss SERI project Compression and Evaluation of High Dynamic Range Image and Video (C12.0081), Portuguese Instituto de Telecomunicações - "FCT - Fundação para a Ciência e a Tecnologia" (project UID/EEA/50008/2013), and COST IC1005 The digital capture, storage, transmission, and display of real-world lighting HDRi.

\section{Author details}

${ }^{1}$ Multimedia Signal Processing Group, EPFL, Lausanne, Switzerland. ${ }^{2}$ Optics Center, UBI, Covilhã, Portugal. ${ }^{3}$ Instituto de Telecomunicações, UBI, Covilhã, Portugal.

Received: 4 May 2015 Accepted: 3 November 2015

Published online: 02 December 2015

References

1. P Hanhart, P Korshunov, T Ebrahimi, in SPIE Applications of Digital Image Processing XXXVII. Subjective evaluation of higher dynamic range video, (2014)

2. P Korshunov, H Nemoto, A Skodras, T Ebrahimi, in Proc. SPIE 9138, Optics, Photonics, and Digital Technologies for Multimedia Applications III. Crowdsourcing-based Evaluation of Privacy in HDR Images, (2014)

3. E Reinhard, G Ward, S Pattanaik, P Debevec, High Dynamic Range Imaging: Acquisition, Display, and Image-Based Lighting (The Morgan Kaufmann Series in Computer Graphics). (Morgan Kaufmann Publishers Inc., San Francisco, CA, USA, 2005)

4. H Seetzen, W Heidrich, W Stuerzlinger, G Ward, L Whitehead, M Trentacoste, A Ghosh, A Vorozcovs, High Dynamic Range Display Systems. ACM Trans. Graph. 23(3), 760-768 (2004)

5. E Reinhard, M Stark, P Shirley, J Ferwerda, Photographic tone reproduction for digital images. ACM Trans. Graph. 21(3), 267-276 (2002) 
6. TRichter, in Picture Coding Symposium. On the standardization of the JPEG XT image compression, (2013)

7. G Ward, M Simmons, in ACM SIGGRAPH 2006 Courses. JPEG-HDR: a backwards-compatible, high dynamic range extension to JPEG, (2006)

8. TRichter, in SPIE Applications Of Digital Image Processing XXXVII. On the integer coding profile of JPEG XT, vol. 9217, (2014)

9. P Hanhart, M Rerabek, T Ebrahimi, in Proc. SPIE, Applications of Digital Image Processing XXXVIII. Towards high dynamic range extensions of HEVC: subjective evaluation of potential coding technologies, (2015)

10. R Mantiuk, S Daly, K Myszkowski, H-P Seidel, in SPIE Human Vision and Electronic Imaging $X$, vol. 5666. Predicting visible differences in high dynamic range images: model and its calibration, (2005)

11. SJ Daly, in SPIE Human Vision, Visual Processing, and Digital Display III, vol. 1666. Visible differences predictor: an algorithm for the assessment of image fidelity, (1992)

12. R Mantiuk, KJ Kim, AG Rempel, W Heidrich, HDR-VDP-2: A calibrated visual metric for visibility and quality predictions in all luminance conditions. ACM Trans. Graph. 30(4), 40:1-40:14 (2011)

13. M Narwaria, RK Mantiuk, M Perreira Da Silva, P Le Callet, HDR-VDP-2.2: a calibrated method for objective quality prediction of high-dynamic range and standard images. J. Electron. Imaging. 24(1), 010501 (2015)

14. TO Aydin, R Mantiuk, K Myszkowski, H-P Seidel, Dynamic Range Independent Image Quality Assessment. ACM Trans. Graph. 27(3), 69:1-69:10 (2008)

15. M Narwaria, M Perreira Da Silva, P Le Callet, HDR-VQM: An objective quality measure for high dynamic range video. Signal Process. Image Commun. 35, 46-60 (2015)

16. C Poynton, Digital Video and HD: Algorithms and Interfaces. (Elsevier/Morgan Kaufmann, Burlington, Vermont, USA, 2012)

17. SK Shevell, The Science of Color. (Elsevier, Boston, Massachusetts, USA, 2003)

18. A Rose, The sensitivity performance of the human eye on an absolute scale. J. Opt. Soc. Am. A. 38(2), 196-208 (1948)

19. H De Vries, The quantum character of light and its bearing upon threshold of vision, the differential sensitivity and visual acuity of the eye. Physica. 10(7), 553-564 (1943)

20. TO Aydın, R Mantiuk, K Myszkowski, H-P Seidel, in SPIE Human Vision and Electronic Imaging XIII, vol. 6806. Extending quality metrics to full luminance range images, (2008)

21. J Munkberg, P Clarberg, J Hasselgren, T Akenine-Möller, High Dynamic Range Texture Compression for Graphics Hardware. ACM Trans. Graph. 25(3), 698-706 (2006)

22. HR Sheikh, MF Sabir, AC Bovik, A Statistical Evaluation of Recent Full Reference Image Quality Assessment Algorithms. IEEE Trans. Image Process. 15(11), 3440-3451 (2006)

23. K Seshadrinathan, R Soundararajan, AC Bovik, LK Cormack, Study of Subjective and Objective Quality Assessment of Video. IEEE Trans. Image Process. 19(6), 1427-1441 (2010)

24. G Valenzise, F De Simone, P Lauga, F Dufaux, in SPIE Applications of Digital Image Processing XXXVII, vol. 9217. Performance evaluation of objective quality metrics for HDR image compression, (2014)

25. C Mantel, SC Ferchiu, S Forchhammer, in 16th International Workshop on Multimedia Signal Processing. Comparing subjective and objective quality assessment of HDR images compressed with JPEG-XT, (2014)

26. P Hanhart, MV Bernardo, P Korshunov, M Pereira, AMG Pinheiro, T Ebrahimi, in 6th International Workshop on Quality of Multimedia Experience. HDR image compression: A new challenge for objective quality metrics, (2014)

27. M Azimi, A Banitalebi-Dehkordi, Y Dong, MT Pourazad, P Nasiopoulos, in International Conference on Multimedia Signal Processing. Evaluating the Performance of Existing Full-Reference Quality Metrics on High Dynamic Range (HDR) Video Content, (2014)

28. M Rerabek, P Hanhart, P Korshunov, T Ebrahimi, in 9th International Workshop on Video Processing and Quality Metrics for Consumer Electronics. Subjective and objective evaluation of HDR video compression, (2015)

29. P Korshunov, P Hanhart, T Ricther, A Artusi, R Mantiuk, T Ebrahimi, in 7th International Workshop on Quality of Multimedia Experience. Subjective quality assessment database of HDR images compressed with JPEG XT, (2015)

30. Subjective Quality Assessment Database of HDR Images Compressed with JPEG XT. http://mmspg.epfl.ch/jpegxt-hdr. Accessed 23 July 2015
31. S Miller, M Nezamabadi, S Daly, Perceptual signal coding for more efficient usage of bit codes. SMPTE Motion Imaging J. 122(4), 52-59 (2013)

32. R Mantiuk, S Daly, L Kerofsky, Display adaptive tone mapping. ACM Trans. Graph. 27(3), 68 (2008)

33. AO Akyüz, E Reinhard, Color appearance in high-dynamic-range imaging SPIE J. Electron. Imaging. 15(3) (2006)

34. ITU-R BT.2022, General viewing conditions for subjective assessment of quality of SDTV and HDTV television pictures on flat panel displays. International Telecommunication Union (2012)

35. ITU-R BT.500-13, Methodology for the subjective assessment of the quality of television pictures. International Telecommunication Union (2012)

36. V Laparra, JM noz-Marí, J Malo, Divisive normalization image quality metric revisited. J. Opt. Soc. Am. A. 27(4), 852-864 (2010)

37. Z Wang, AC Bovik, A universal image quality index. IEEE Signal Process. Lett. 9(3), 81-84 (2002)

38. Z Wang, AC Bovik, HR Sheikh, EP Simoncelli, Image quality assessment: from error visibility to structural similarity. IEEE Trans. Image Process. 13(4), 600-612 (2004)

39. Z Wang, EP Simoncelli, AC Bovik, in 37th Asilomar Conference on Signals, Systems and Computers. Multiscale structural similarity for image quality assessment, (2003)

40. A Shnayderman, A Gusev, AM Eskicioglu, An SVD - based grayscale image quality measure for local and global assessment. IEEE Trans. Image Process. 15(2), 422-429 (2006)

41. S Aja-Fernandéz, RSJ Estepar, C Alberola-Lopez, C-F Westin, in 28th Annual International Conference of the IEEE Engineering in Medicine and Biology Society. Image Quality Assessment based on Local Variance, (2006)

42. HR Sheikh, AC Bovik, G de Veciana, An information fidelity criterion for image quality assessment using natural scene statistics. IEEE Trans. Image Process. 14(12), 2117-2128 (2005)

43. HR Sheikh, AC Bovik, Image information and visual quality. IEEE Trans. Image Process. 15(2), 430-444 (2006)

44. L Zhang, D Zhang, X Mou, D Zhang, FSIM: A feature similarity index for image quality assessment. IEEE Trans. Image Process. 20(8), 2378-2386 (2011)

45. Z Wang, Q Li, Information Content Weighting for Perceptual Image Quality Assessment. IEEE Trans. Image Process. 20(5), 1185-1198 (2011)

46. XK Yang, WS Ling, ZK Lu, EP Ong, SS Yao, Just noticeable distortion model and its applications in video coding. Signal Process. Image Commun. 20(7), 662-680 (2005)

47. J Mannos, DJ Sakrison, The effects of a visual fidelity criterion of the encoding of images. IEEE Trans. Inf. Theory. 20(4), 525-536 (1974)

48. T Mitsa, KL Varkur, in IEEE International Conference on Acoustics, Speech, and Signal Processing. Evaluation of contrast sensitivity functions for the formulation of quality measures incorporated in halftoning algorithms, (1993)

49. CIE, Colorimetry Official Recommendation of the International Commission on Illumination. CIE publication 15.2, CIE Central Bureau (1986)

50. CIE, Industrial Colour-Difference Evaluation. CIE publication 116, CIE Central Bureau (1995)

51. FJJ Clarke, R McDonald, B Rigg, Modification to the JPC79 Colour-difference Formula. J. Soc. Dye. Colour. 100(4), 128-132 (1984)

52. M Luo, G Cui, B Rigg, The development of the CIE 2000 colour-difference formula: CIEDE2000. Color Res. Appl. 26(5), 340-350 (2001)

53. SJ Erasmus, KCA Smith, An automatic focusing and astigmatism correction system for the SEM and CTEM. J. Microsc. 127(2), 185-199 (1982)

54. CF Batten, Autofocusing and astigmatism correction in the scanning electron microscope. Master's thesis. (University of Cambridge, UK, 2000)

55. AV Murthy, $\amalg$ Karam, in 2nd International Workshop on Quality of Multimedia Experience. A MATLAB-based framework for image and video quality evaluation, (2010)

56. D Shaked, I Tastl, in IEEE International Conference on Image Processing. Sharpness measure: towards automatic image enhancement, (2005)

57. N Zhang, A Vladar, M Postek, B Larrabee, in Proceedings Section of Physical and Engineering Sciences of American Statistical Society. A kurtosis-based statistitcal measure for two-dimensional processes and its application to image sharpness, (2003)

58. R Ferzli, LJ Karam, J Caviedes, in 1st International Workshop on Video Processing and Quality Metrics for Consumer Electronics. A robust image sharpness metric based on kurtosis measurement of wavelet coefficients, (2005) 
59. R Ferzli, L Karam, in 3rd International Workshop on Video Processing and Quality Metrics for Consumer Electronics. A no-reference objective sharpness metric using riemannian tensor, (2007)

60. H Wilson, A transducer function for threshold and suprathreshold human vision. Biol. Cybern. 38(3), 171-178 (1980)

61. PG Barten, Contrast Sensitivity of the Human Eye and Its Effects on Image Quality. (SPIE Optical Engineering Press, Bellingham, Washington, USA, 1999)

62. ITU-R BT.709, Parameter values for the HDTV standards for production and international programme exchange. International

Telecommunication Union (2002)

63. P Hanhart, P Korshunov, T Ebrahimi, in 18th International Conference on Digital Signal Processing. Benchmarking of quality metrics on ultra-high definition video sequences, (2013)

64. ITU-T P.1401, Methods, metrics and procedures for statistical evaluation, qualification and comparison of objective quality prediction models. International Telecommunication Union (2012)

65. Q Huynh-Thu, M Ghanbari, Scope of validity of PSNR in image/video quality assessment. Electron. Lett. 44(13), 800-801 (2008)

\section{Submit your manuscript to a SpringerOpen ${ }^{\circ}$ journal and benefit from:}

- Convenient online submission

- Rigorous peer review

- Immediate publication on acceptance

- Open access: articles freely available online

- High visibility within the field

- Retaining the copyright to your article

Submit your next manuscript at $\gg$ springeropen.com 